\title{
Modified exponential function method for nonlinear mathematical models with Atangana conformable derivative
}

\author{
T. Aktürk \\ Department of Mathematics and Science Education, Ordu University, 52100 Ordu/Turkey. \\ e-mail: tolgaakturkk@gmail.com
}

Received 5 February 2021; accepted 10 March 2021

In this study, we investigate the exact solutions of the modifie Benjamin-Bona-Mahony and Sharma-Tasso-Olver equations, which are define with Atangana conformable fractional derivative, using the modifie exponential function method. Exact solutions of the modifie Benjamin-Bona-Mahony and Sharma-Tasso-Olver equations were obtained by using the modifie exponential function method. Two and three-dimensional and contour graphics are used to understand the physical interpretations of the resulting exact solutions to the mathematical model. When all these results and graphs are analyzed, it has been shown that the modifie exponential function method is an effective method for obtaining exact solutions for all other nonlinear fractional partial differential equations containing conformable fractional derivatives of Atangana.

Keywords: The modifie exponential function method; The space-time fractional modifie Benjamin-Bona-Mahony equation; Fractional Sharma-Tasso-Olver equation; Atangana conformable derivative; contour surfaces.

PACS: 02.30.Jr; 02.60.Cb; 04.20.Jb

DOI: https://doi.org/10.31349/RevMexFis.67.040704

\section{Introduction}

Nonlinear partial differential equations are used in various field of physics, flui mechanics, engineering, health, etc. These equations are widely used for its applications in mathematical modeling of situations encountered across many areas. It is very important to obtain numerical and exact solutions to such mathematical models because with the analysis of the solutions of such mathematical models, a physical interpretation of the situation it represents can be made. For example, in the context of the COVID-19 outbreak, mathematical models have allowed us to give notice of the extent of the epidemic and to measure the rate of spread of the virus, among other useful information; these tools are, and will continue to be, developed in the future. However, there are various methods in the literature about the investigation of the solutions of nonlinear partial differential equations [18]. In recent years, intensive studies have been carried out in the scientifi world to investigate fractional partial differential equations compatible with nonlinear partial differential equations and their solutions. Some fractional derivative operators and nonlinear fractional differential equations have been introduced in the literature [9-24].

A new fractional derivative operator, which is arranged as a fractional derivative, has been determined, and using this definition the exact solutions of the nonlinear fractional differential equation have been obtained [25]. Atangana et al., while giving place to some theorems and properties about conformable derivative, they named this term as beta derivative [26]. The space-time fractional modifie BenjaminBona-Mahony and fractional Sharma-Tasso-Olver equations given with Atanagana's conformable derivative were solved by the firs integral method [27].

In this article, exact solutions of nonlinear fractional differential equations were investigated with the use of the modifie exponential function method.

\section{Beta-derivatives}

Definition 1. A new fractional derivative called conformable derivative by Khalil et al. has been brought to the literature [25]. Let, the function $f:[0, \infty)$ of the $\alpha$-th order analyzed as the conformable derivative function is as follows in terms of $t>0, \alpha \in(0,1)$ :

$$
{ }_{0} D_{t}^{\alpha}\{f(t)\}=\lim _{\varepsilon \rightarrow 0} \frac{f\left(t+\varepsilon t^{1-\alpha}\right)-f(t)}{\varepsilon} .
$$

When $f$ is $\alpha$-differentiable in the range of $(0, a), a>0$ and $\lim _{\varepsilon \rightarrow 0^{+}} f^{(\alpha)}(t)$ exists, then it can be stated as $f^{(\alpha)}(0)=$ $\lim _{\varepsilon \rightarrow 0^{+}} f^{(\alpha)}(t)$.

Definition 2. The beta derivative stated by Atangana et al. is define as follows [26]:

$$
{ }_{0}^{A} D_{t}^{\alpha}\{f(t)\}=\lim _{\varepsilon \rightarrow 0} \frac{f\left(t+\varepsilon\left[t+\frac{1}{\Gamma(\alpha)}\right]^{1-\alpha}\right)-f(t)}{\varepsilon} .
$$

The most important reason for choosing the fractional derivative of Atangana is that the basic derivatives can provide some useful properties. Some features of the definitio given above are stated below:

i) Let $g \neq 0$ and $f$ functions be differentiable with respect to beta in the interval $\beta \in(0,1]$. In this case, the following equation can be written that can be provided for all real numbers $a$ and $b$. 
${ }_{0}^{A} D_{x}^{\alpha}\{a f(x)+b g(x)\}=a_{0}^{A} D_{x}^{\alpha}\{f(x)\}+b_{0}^{A} D_{x}^{\alpha}\{g(x)\}$.

ii) Where $c$ is any constant that satisfie the following equation,

$$
{ }_{0}^{A} D_{x}^{\alpha}\{f(t)\}=0
$$

iii)

$$
\begin{gathered}
{ }_{0}^{A} D_{x}^{\alpha}\{f(x) g(x)\}=g(x)_{0}^{A} D_{x}^{\alpha}\{f(x)\} \\
+f(x)_{0}^{A} D_{x}^{\alpha}\{g(x)\} .
\end{gathered}
$$

iv)

$$
{ }_{0}^{A} D_{x}^{\alpha}\left\{\frac{f(x)}{g(x)}\right\}=\frac{g(x)_{0}^{A} D_{x}^{\alpha}\{f(x)\}+f(x)_{0}^{A} D_{x}^{\alpha}\{g(x)\}}{g^{2}(x)}
$$

If $\varepsilon=(x+1 / \Gamma(\alpha))^{\alpha-1} h$ is written instead of $\varepsilon$ in Eq. (2) and $h \rightarrow 0$, when $\varepsilon \rightarrow 0$, we get as follows,

$$
{ }_{0}^{A} D_{x}^{\alpha}\{f(x)\}=\left(x+\frac{1}{\Gamma(\alpha)}\right)^{1-\alpha} \frac{d f(x)}{d x},
$$

with

$$
\eta=\frac{\delta}{\alpha}\left(x+\frac{1}{\Gamma(\alpha)}\right)^{\alpha}
$$

$\delta$ is constant and in this case the following equation can be written

$$
{ }_{0}^{A} D_{x}^{\alpha}\{f(\eta)\}=\delta \frac{d f(\eta)}{d \eta}
$$

\section{The modified exponential function method}

In this section, exact solutions of nonlinear fractional partial differential equations define as Atangana derivatives will be introduced in detail by using the modifie exponential function method [28].

The general form of the nonlinear fractional partial differential equation containing the two variables $u$ function and the beta derivative is given as follows:

$$
P\left(u,{ }_{0}^{A} D_{x}^{\alpha} u, u_{x}, u_{x x}, \ldots\right)=0,
$$

where given $x$ is space and $t$ is time.

The processing steps of the modifie exponential function are as follows:

Step 1. First of all, we can consider the traveling wave transformation to obtain the wave solution of Eq. (10) as follows:

$$
u(x, t)=u(\eta), \quad \eta=k x-\frac{\lambda}{\alpha}\left(t+\frac{1}{\Gamma(\alpha)}\right)^{\alpha},
$$

where $k$ and $\lambda$ are constants. When the derivative terms required in Eq. (10) are taken from Eq. (11) and replaced, the following nonlinear partial differential equation is obtained,

$$
N\left(u, u^{\prime}, u^{\prime \prime}, u^{\prime \prime \prime}, \ldots\right)=0 .
$$

Step 2. Let, the exact solution of the nonlinear fractional differential equation analyzed in the article be in the form of (13):

$$
\begin{aligned}
u(\eta) & =\frac{\sum_{j=0}^{n} A_{j}\left[e^{-\vartheta(\eta)}\right]^{j}}{\sum_{i=0}^{m}\left[e^{-\vartheta(\eta)}\right]^{i}} \\
& =\frac{A_{0}+A_{1} e^{-\vartheta}+\ldots+A_{n} e^{-n \vartheta}}{B_{0}+B_{1} e^{-\vartheta}+\ldots+B_{m} e^{-m \vartheta}},
\end{aligned}
$$

where $A_{j}, B_{i},(0 \leq j \leq n, 0 \leq i \leq m)$ are constants.

$$
\vartheta^{\prime}(\eta)=e^{-\vartheta(\eta)}+k e^{\vartheta(\eta)}+\sigma .
$$

When Eq. (14) is integrated according to $\eta$ the following solution families are obtained [26]:

Family 1. If $\mu \neq 0$ and $\sigma^{2}-4 \mu>0$,

$$
\begin{aligned}
\vartheta(\eta) & =\ln \left(\frac{-\sqrt{\sigma^{2}-4 \mu}}{2 \mu} \tanh \right. \\
& \left.\times\left[\frac{-\sqrt{\sigma^{2}-4 \mu}}{2}\{\eta+E\}\right]-\frac{\sigma}{2 \mu}\right) .
\end{aligned}
$$

Family 2. If $\mu \neq 0$ and $\sigma^{2}-4 \mu<0$,

$$
\begin{aligned}
\vartheta(\eta) & =\ln \left(\frac{\sqrt{-\sigma^{2}+4 \mu}}{2 \mu} \tan \right. \\
& \left.\times\left[\frac{\sqrt{-\sigma^{2}+4 \mu}}{2}\{\eta+E\}\right]-\frac{\sigma}{2 \mu}\right) .
\end{aligned}
$$

Family 3. If $\mu=0, \sigma \neq 0$ and $\sigma^{2}-4 \mu>0$,

$$
\vartheta(\eta)=\ln \left(\frac{\sigma}{e^{\sigma(\eta+E)}-1}\right) .
$$

Family 4. If $\mu \neq 0, \sigma \neq 0$ and $\sigma^{2}-4 \mu=0$,

$$
\vartheta(\eta)=\ln \left(-\frac{2 \sigma(\eta+E)+4}{\sigma^{2}(\eta+E)}\right) .
$$

Family 5. If $\mu=0, \sigma=0$ and $\sigma^{2}-4 \mu=0$,

$$
\vartheta(\eta)=\ln (\eta+E),
$$

where $E, \sigma, \mu$ are coefficients

Step 3. In this section, the limits of the total symbols should be determined by applying the balance procedure to Eq. (12), which is analyzed as the exact solution of the nonlinear fractional partial differential equation. After the balance procedure is reduced to the form of Eq. (12) under investigation, 
a relation is obtained between $m$ and $n$ by equating the term with the highest order derivative and the highest order nonlinear term. Then, by determining an arbitrary constant to the value of $m$ in this relation, the constant $n$ is obtained. Then, Eq. (13) and its derivatives are written in Eq. (12) and the algebraic equation system containing $e^{-\vartheta(\eta)}$ is get. With the solution of this system, it will be found in constants such as $A_{0}, A_{1}, A_{2}, \ldots, A_{n}, B_{0}, B_{1}, B_{2}, \ldots, B_{m}$. By replacing all these terms, it is checked that they provide the equation and the exact solution function of Eq. (10) is obtained.

\section{Applications of nonlinear fractional differ- ential equations with Atangana derivatives}

In this section, we investigate exact solutions of the SharmaTasso-Olver equation and modifie Benjamin-Bona-Mahony equation equations using the modifie exponential function method with Atangana's conformable derivative.

Example 1. Let's investigate the Sharma-Tasso-Olver equation together with the conformable derivatives of Atangana [27],

$$
\begin{aligned}
{ }_{0}^{A} D_{x}^{\alpha}\{u\} & +3 a\left(u_{x}\right)^{2}+3 a u^{2} u_{x} \\
& +3 a u u_{x x}+a u_{x x x}=0, \quad 0<\alpha \leq 1 .
\end{aligned}
$$

The equation given above is subject to the following initial conditions

$$
u(x, 0)=-\sqrt{2 B_{0}} \tan \left(\frac{\sqrt{2 B_{0}}}{2} x\right) .
$$

where $a$ and $B_{0}$ are arbitrary constants and $\alpha$ is a parameter representing the order of the Atangana derivative. We use the following wave transformation to reduce the Eq. (20) to the nonlinear ordinary differential equation form

$$
\begin{aligned}
u(x, t) & =u(\eta) \quad \text { and } \\
\eta & =x-\frac{\lambda}{\alpha}\left(t+\frac{1}{\Gamma(\alpha)}\right)^{\alpha},
\end{aligned}
$$

where $\lambda$ is constant. Then, we substitute Eq. (22) into Eq. (20), we obtain the following nonlinear ordinary differential equation

$$
-\lambda u^{\prime}+3 a\left(u^{\prime}\right)^{2}+3 a u^{2} u^{\prime}+3 a u u^{\prime \prime}+a u^{\prime \prime \prime}=0 .
$$

Integrating Eq. (23) with respect to $\eta$, we get

$$
c-\lambda u+3 a u u^{\prime}+a u^{3}+a u^{\prime \prime}=0,
$$

where $c$ is integral constant.

According to the balance procedure, using the highest order nonlinear term $u^{3}$ and the highest order term $u^{\prime \prime}$ in Eq. (24), the following equation is obtained

$$
3 n-3 m=n-m+2 \Rightarrow n=m+1 \text {. }
$$

If $m=1$, we then get $n=2$.

In this case, Eq. (13) is written as follows.

$$
U(\eta)=\frac{\gamma}{\phi}=\frac{a_{0}+a_{1} e^{-\vartheta}+A_{2} e^{-2 \vartheta}}{B_{0}+B_{1} e^{-\vartheta}}
$$

Let us obtain the necessary derivative terms in Eq. (24) from Eq. (26),

$$
\begin{aligned}
u^{\prime}(\eta) & =\frac{\gamma^{\prime} \phi-\gamma \phi^{\prime}}{\phi^{2}} \\
u^{\prime \prime}(\eta) & =\frac{\left(\left[\gamma^{\prime \prime} \phi^{3}+\gamma^{\prime} \phi^{\prime} \phi^{2}-\left\{\phi^{2} \gamma^{\prime} \phi^{\prime}+\phi^{2} \gamma \phi^{\prime \prime}\right\}\right]-2 \phi \phi^{\prime}\left[\gamma^{\prime} \phi-\gamma \phi^{\prime}\right]\right)}{\phi^{4}}
\end{aligned}
$$

If Eqs. (26-28) are substituted in Eq. (24), the exact solutions that satisfy Eq. (20) are as follows:

Case 1.

$$
\begin{aligned}
A_{0} & =\frac{B_{0}^{2}}{A_{2}}, \quad A_{1}=2 B_{0} \quad B_{1}=A_{2}, \\
\mu & =-\frac{\lambda}{a}+\sigma^{2}+\frac{3 B_{0}\left(-\sigma A_{2}+B_{0}\right)}{A_{2}^{2}} \\
c & =\frac{\left(\sigma A_{2}-2 B_{0}\right)\left(\left[\lambda-a \sigma^{2}\right] A_{2}^{2}+4 a \sigma A_{2} B_{0}-4 a B_{0}^{2}\right)}{A_{2}^{3}} .
\end{aligned}
$$

If the coefficient in (29) are replaced in Eq. (26), the exact solution functions of Eq. (20) under the terms of solution families are as follows;

Family 1. When, $\mu \neq 0$ and $\sigma^{2}-4 \mu>0$

$$
u_{1,1}(x, t)=\left(\frac{B_{0}}{A_{2}}-\frac{2 \mu}{\sigma+\sqrt{-4 \mu+\sigma^{2}} \tanh \left[\frac{1}{2} \sqrt{-4 \mu+\sigma^{2}}\{E+\eta\}\right]}\right) .
$$



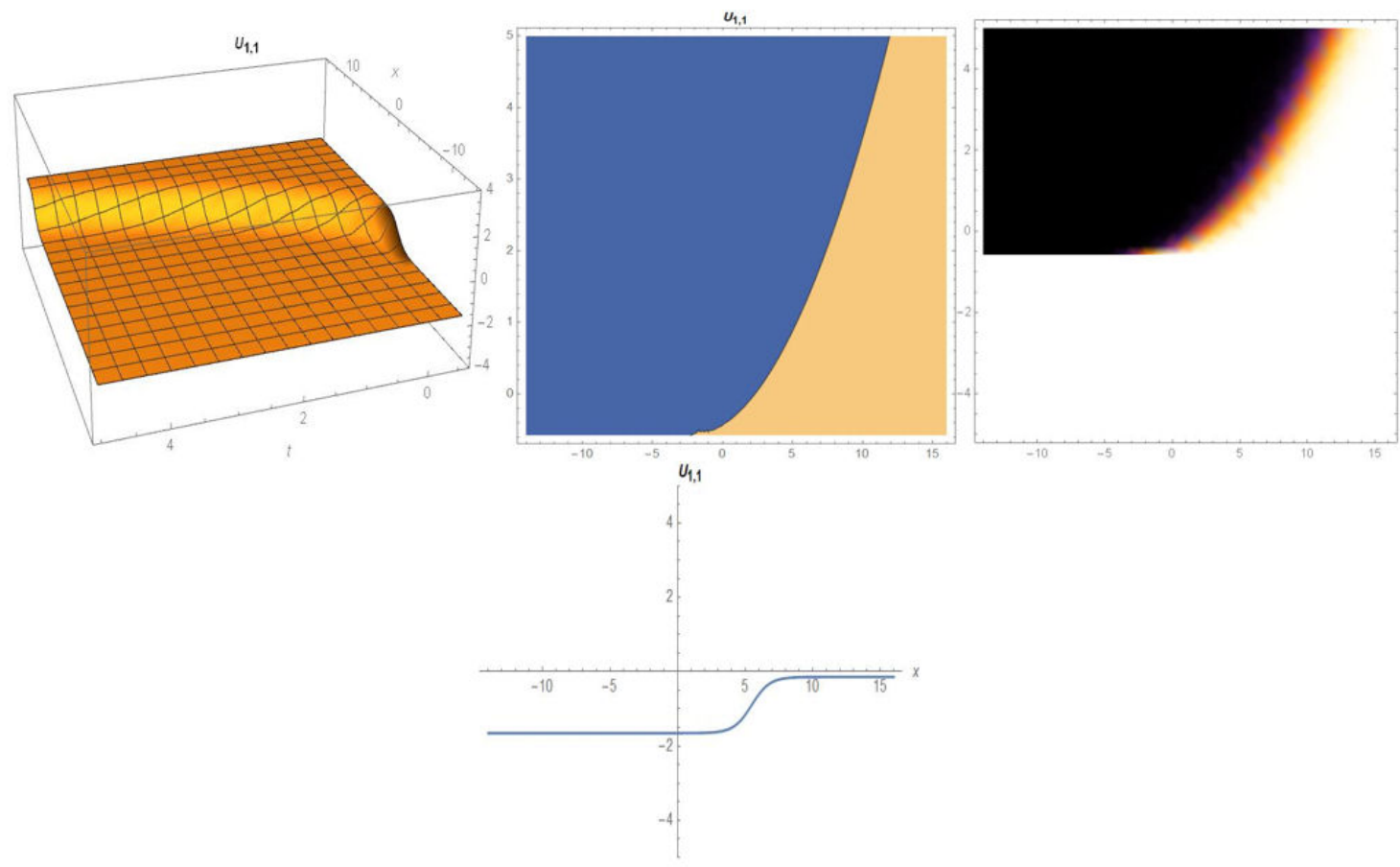

FIGURE 1. Three-dimensional, contour and density plots of the solution (30) for the values $E=0.75, a=1, \alpha=0.5, \lambda=3, c=-0.432$, $\sigma=2, \mu=0.43$, and two-dimensional $t=1$.

Family 2. When, $\mu \neq 0$ and $\sigma^{2}-4 \mu<0$

$$
u_{1,2}(x, t)=\left(\frac{B_{0}}{A_{2}}-\frac{2 \mu}{\sigma-\sqrt{4 \mu-\sigma^{2}} \tan \left[\frac{1}{2} \sqrt{4 \mu+\sigma^{2}}\{E+\eta\}\right]}\right) .
$$
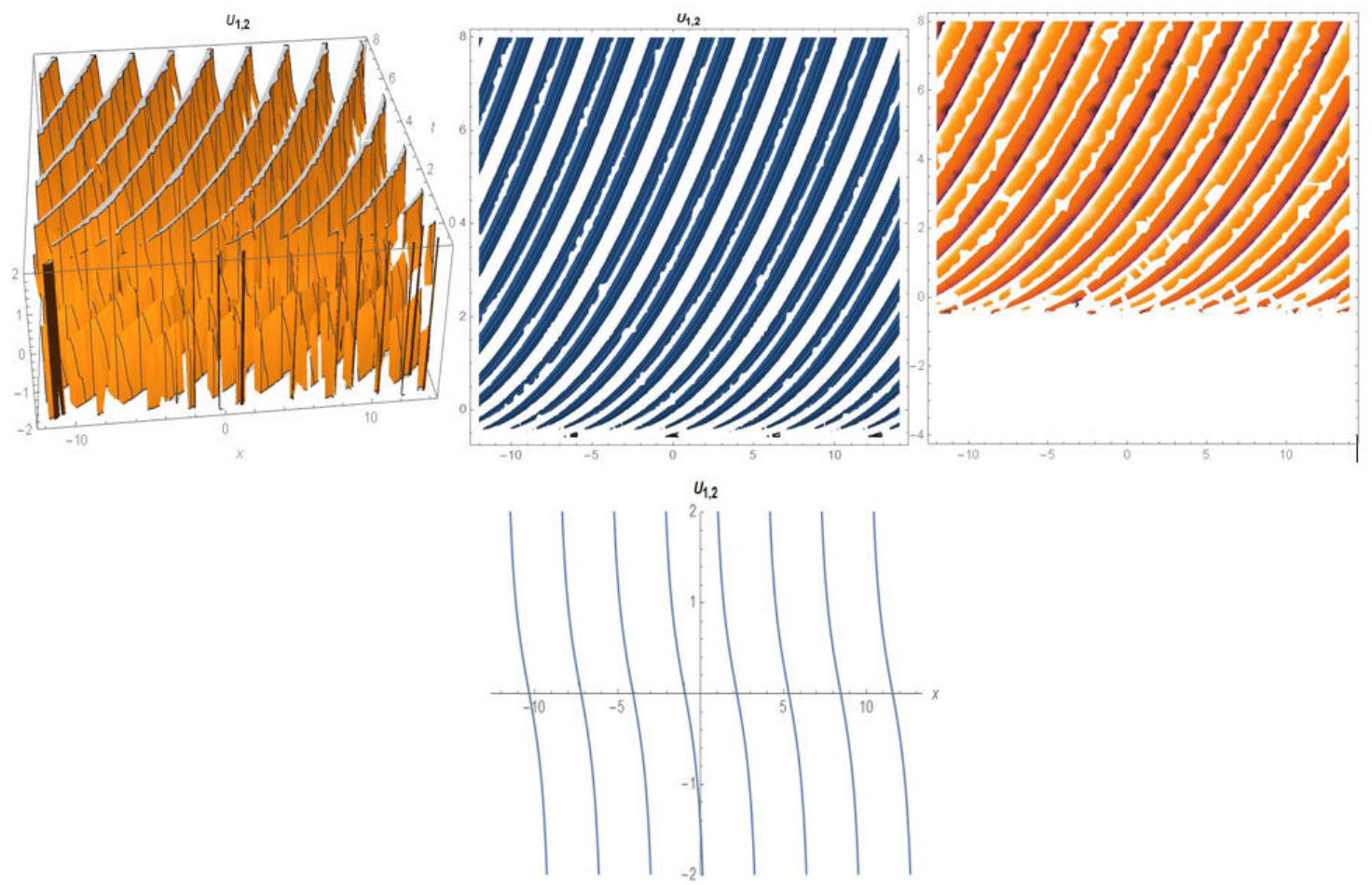

FIGURE 2. Three-dimensional, contour and density plots of the solution (31) for the values $E=0.75, a=1, \alpha=0.5, \lambda=3, c=-0.101$, $\sigma=0.1, \mu=1.01$, and two-dimensional $t=1$. 

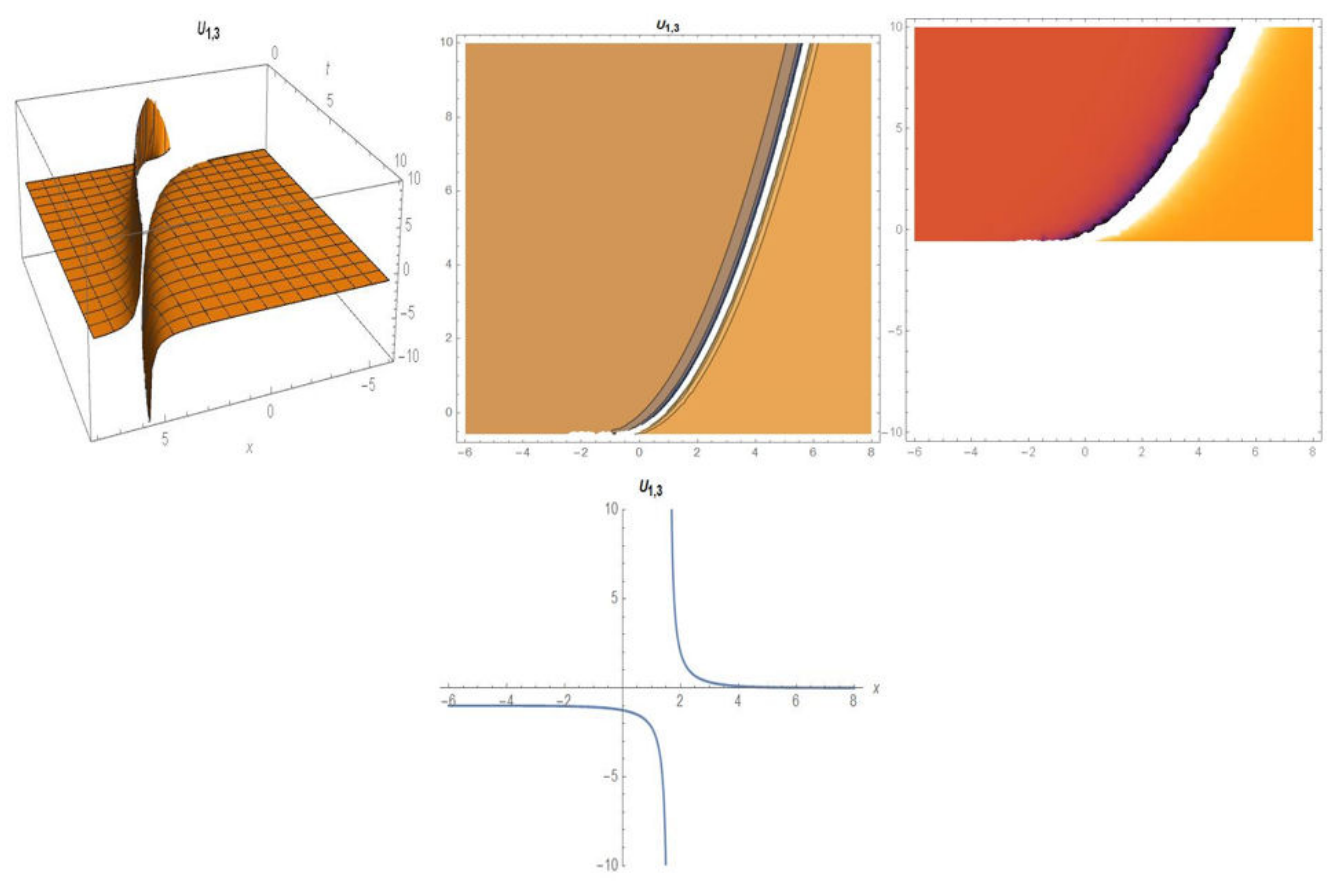

FIGURE 3. Three-dimensional, contour and density plots of the solution (32) for the values $E=0.75, a=1, \alpha=0.5, \lambda=1, c=0$, $\sigma=1, \mu=0$, and two-dimensional $t=0.8$.

Family 3. When, $\mu=0, \sigma \neq 0$ and $\sigma^{2}-4 \mu>0$

$$
u_{1,3}(x, t)=\left(\frac{\lambda}{-1+e^{\sigma(E+\eta)}}+\frac{B_{0}}{A_{2}}\right) .
$$
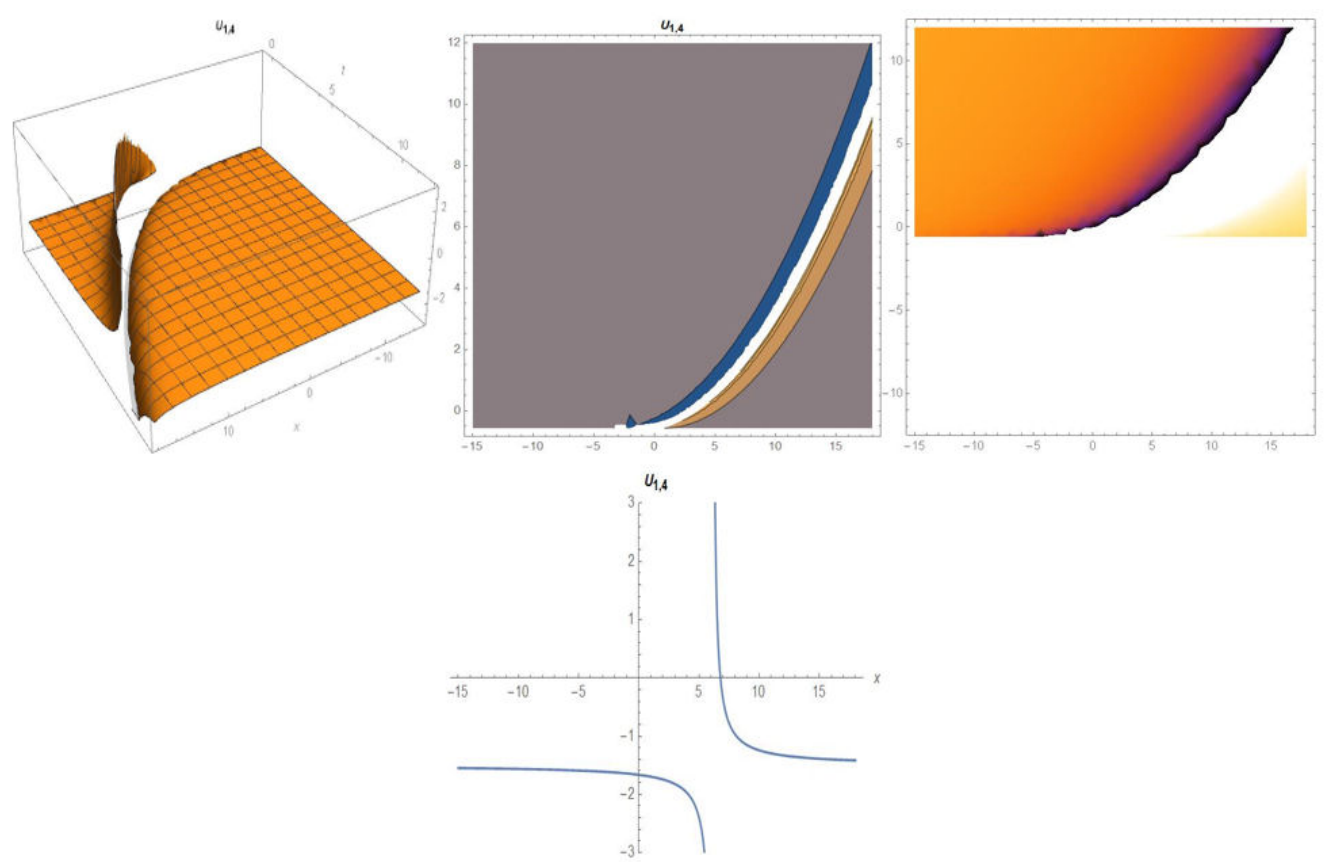

FIGURE 4. Three-dimensional, contour and density plots of the solution (33) for the values $E=0.75, a=1, \alpha=0.5, \lambda=3, c=-2$, $\sigma=2, \mu=1$, and two-dimensional $t=1$.

Family 4. When, $\mu \neq 0, \sigma \neq 0$ and $\sigma^{2}-4 \mu=0$

$$
u_{1,4}(x, t)=\left(\frac{B_{0}}{A_{2}}+\lambda\left[-\frac{1}{2}+\frac{\alpha}{\alpha\{2+(E+x) \lambda\}-\lambda\{x-\eta\}}\right]\right) .
$$



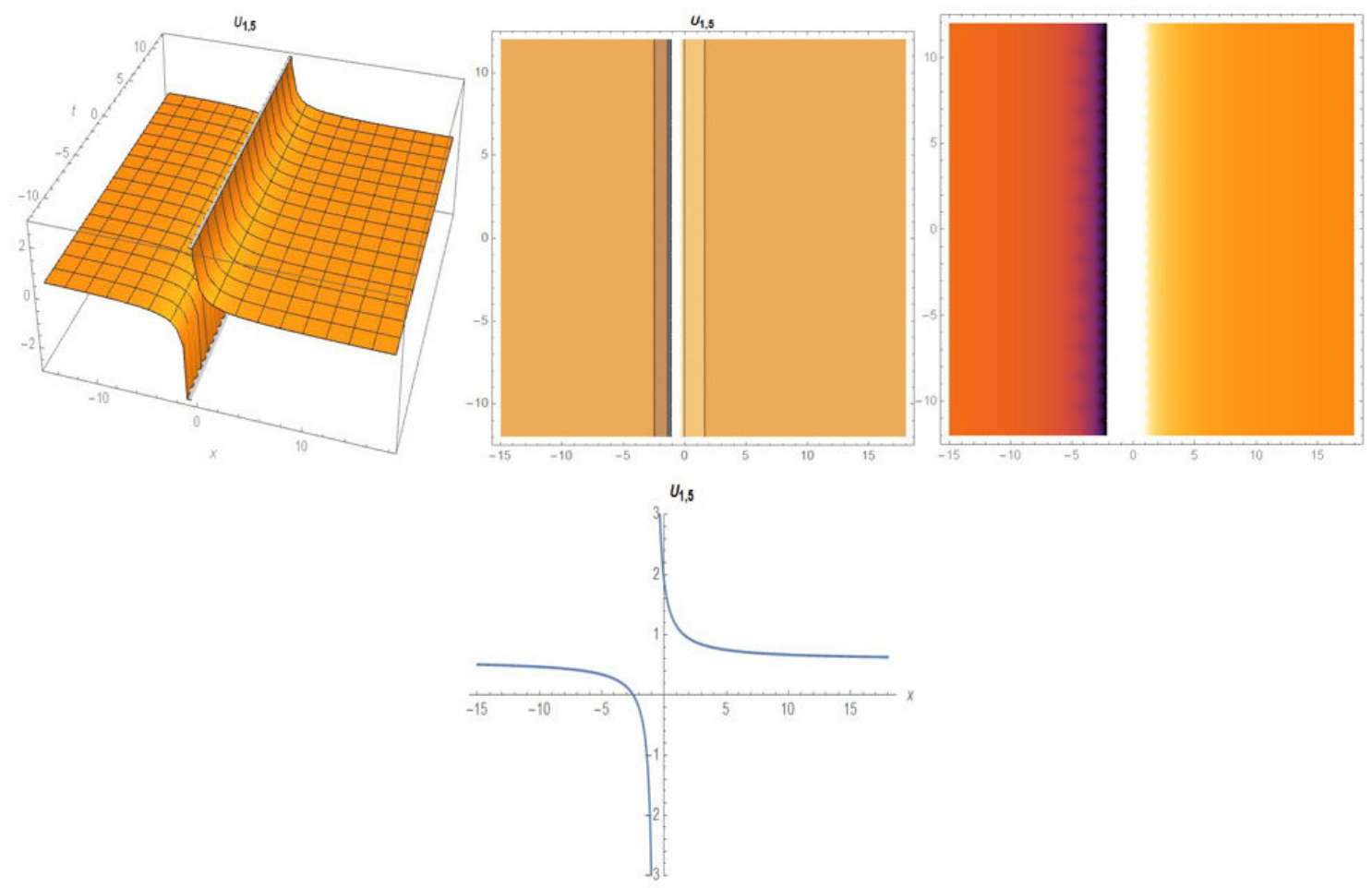

FIGURE 5. Three-dimensional, contour and density plots of the solution (34) for the values $E=0.75, a=1, \alpha=0.5, \lambda=1, c=2 / 3 \sqrt{3}$, $\sigma=0, \mu=0$, and two-dimensional $t=1$.

Family 5. When, $\mu=0, \sigma=0$ and $\sigma^{2}-4 \mu=0$

$$
u_{1,5}(x, t)=\left(\frac{B_{0}}{A_{2}}+\frac{1}{E+x}\right) .
$$

Case 2.

$$
\begin{aligned}
& A_{0}=\frac{B_{0}\left(3 a B_{0}+\sqrt{3 a\left([\lambda+a \mu] A_{2}^{2}-a B_{0}^{2}\right)}\right)}{3 a A_{2}} \quad A_{1}=\frac{6 a B_{0}+\sqrt{3 a\left([\lambda+a \mu] A_{2}^{2}-a B_{0}^{2}\right)}}{3 a} \\
& B_{1}=A_{2}, \quad \sigma=\frac{2 B_{0}}{A_{2}}, \quad c=\frac{2\left([\lambda+4 a \mu] A_{2}^{2}-4 a B_{0}^{2}\right) \sqrt{a\left([\lambda+a \mu] A_{2}^{2}-a B_{0}^{2}\right)}}{3 \sqrt{3} a A_{2}^{3}} .
\end{aligned}
$$

We substitute the coefficient obtained above in Eq. (26). Then, we form the necessary derivative terms for Eq. (20) and write them in their place and obtain exact solution functions under the following solution families.

Family 1. If, $\mu \neq 0$ and $\sigma^{2}-4 \mu>0$

$$
u_{2,1}(x, t)=\left(\frac{([\varsigma \sigma+3 a\{-4 \mu-\sigma\}+\{3 a+\varsigma\} \rho])}{\left(6 a\left[\sigma+\sqrt{-4 \mu+\sigma^{2}} \rho\right]\right)}\right) .
$$

where, $\varsigma=\sqrt{-3 a(-12+a)}$ and $\rho=\tanh \left((1 / 2) \sqrt{-4 \mu+\sigma^{2}}(E+\eta)\right)$.

Family 2. When, $\mu \neq 0$ and $\sigma^{2}-4 \mu<0$

$$
u_{2,2}(x, t)=\left(\frac{\left(\left[\varsigma \sigma+3 a\{-4 \mu-\sigma\}-\{3 a+\varsigma\} \sqrt{4 \mu-\sigma^{2}} \varpi\right]\right)}{\left(6 a\left[\sigma-\sqrt{4 \mu-\sigma^{2}} \varpi\right]\right)}\right) .
$$

where, $\varpi=\tan \left((1 / 2) \sqrt{4 \mu-\sigma^{2}}(E+\eta)\right)$.

Family 3. When, $\mu=0, \sigma \neq 0$ and $\sigma^{2}-4 \mu>0$

$$
u_{2,3}(x, t)=\left(\frac{1}{6}\left[3+\frac{\sqrt{36-3 a}}{\sqrt{a}}+\frac{6 \lambda}{-1+e^{\sigma(E+\eta)}}\right]\right) .
$$



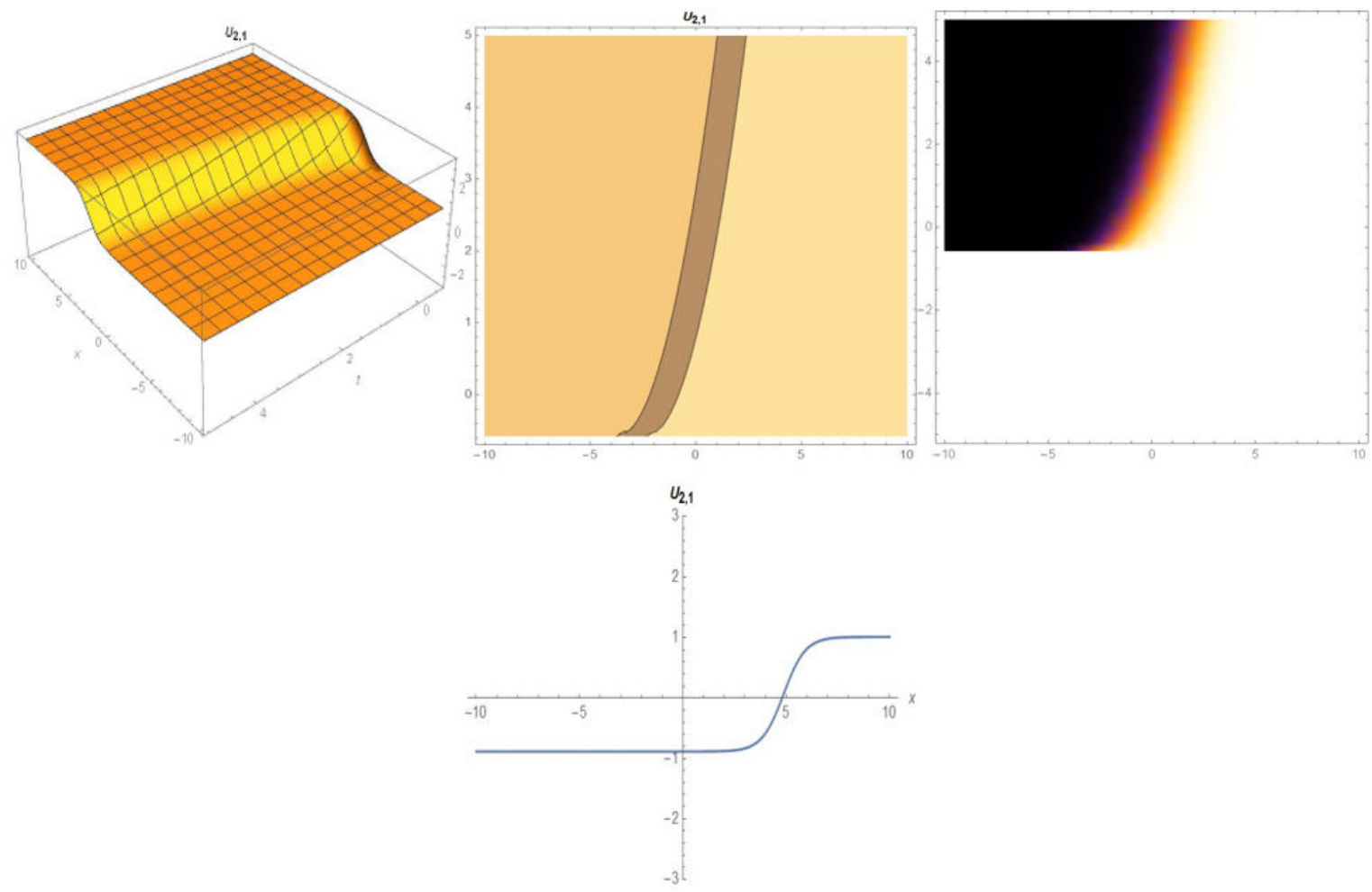

FIgURE 6. Three-dimensional, contour and density plots of the solution (36) for the values $E=0.75, a=0.2, \alpha=0.5, \lambda=1$, $c=0.218222, \sigma=2, \mu=0.43$, and two-dimensional $t=1$.
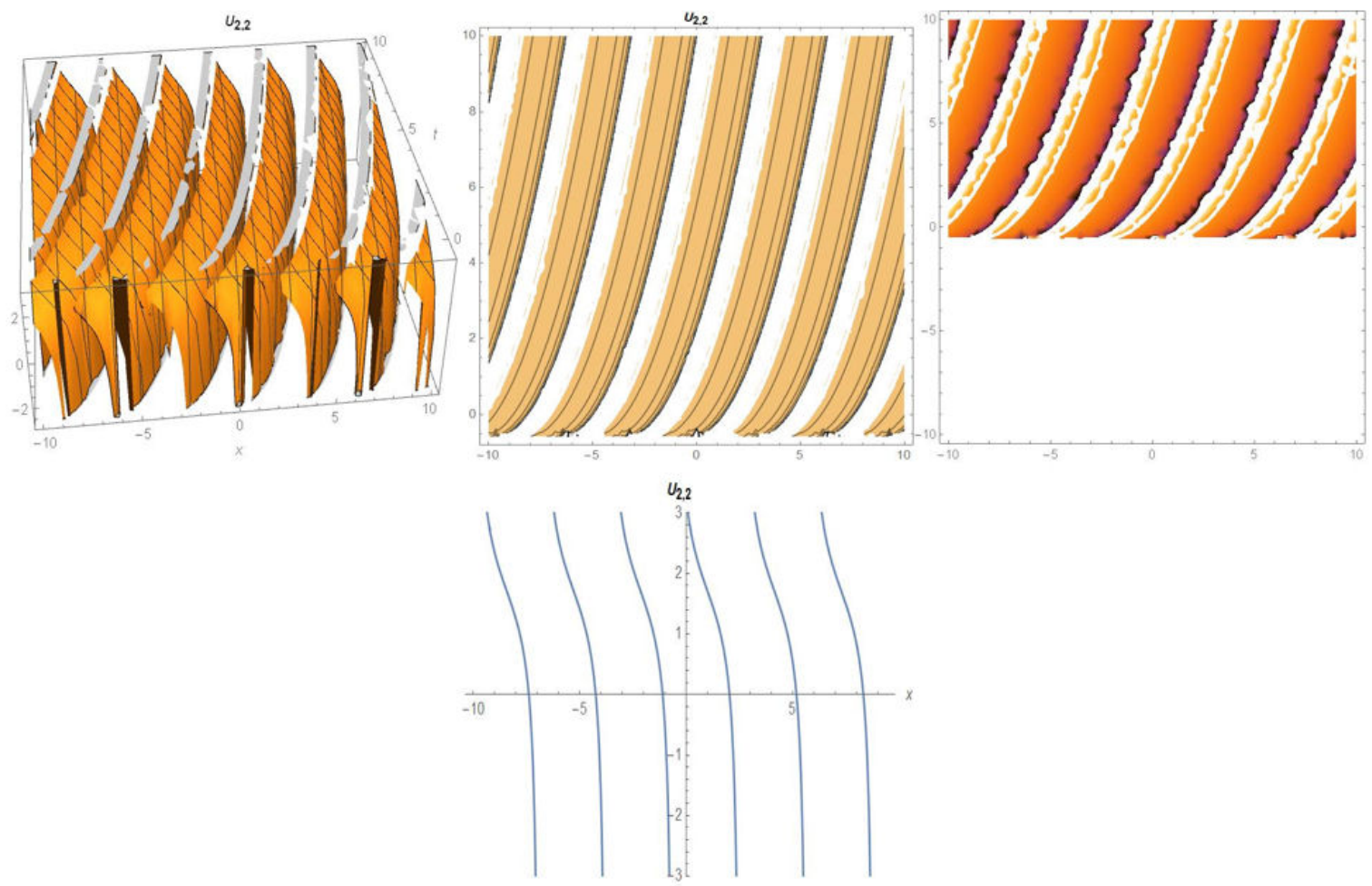

FIgURE 7. Three-dimensional, contour and density plots of the solution (37) for the values $E=0.75, a=0.2, \alpha=0.5, \lambda=1$, $c=1.697056, \sigma=2, \mu=2$, and two-dimensional $t=1$. 

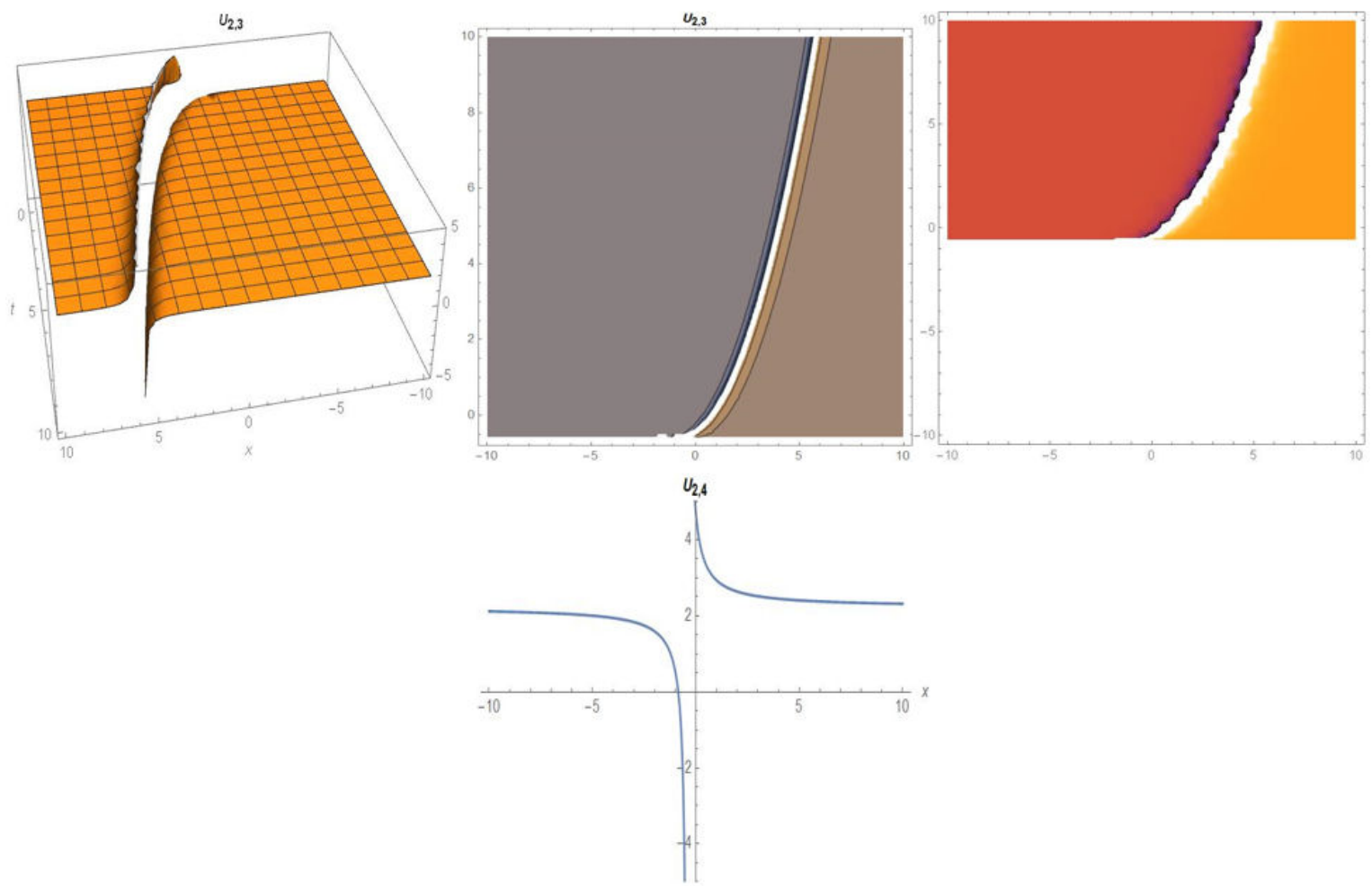

FiguRE 8. Three-dimensional, contour and density plots of the solution (38) for the values $E=0.75, a=0.2, \alpha=0.5, \lambda=1$, $c=0.153960, \sigma=2, \mu=0$, and two-dimensional $t=1$.
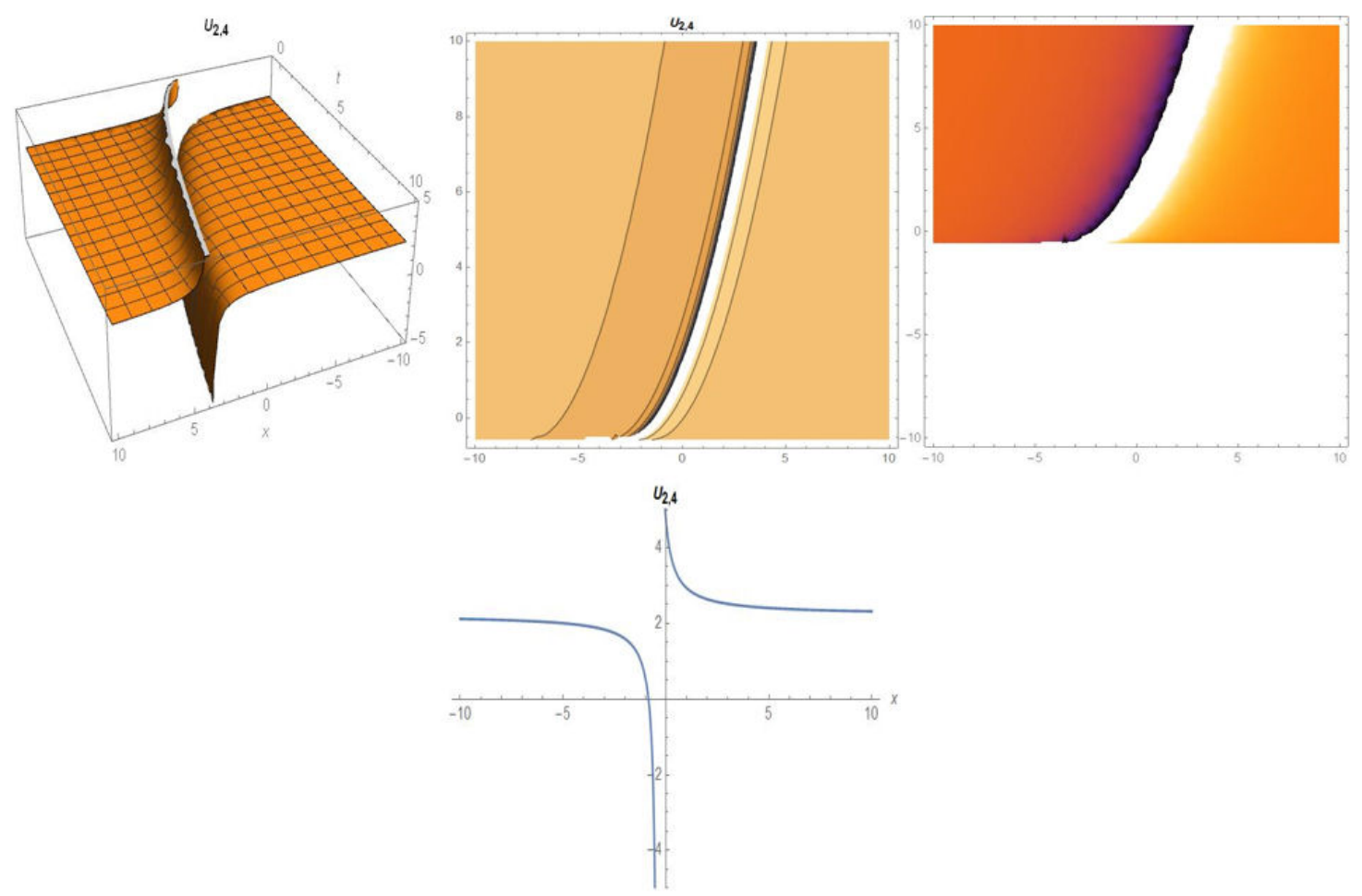

FIgURE 9. Three-dimensional, contour and density plots of the solution (39) for the values $E=0.75, a=0.2, \alpha=0.5, \lambda=1$, $c=0.860662, \sigma=2, \mu=1$, and two-dimensional $t=1$. 

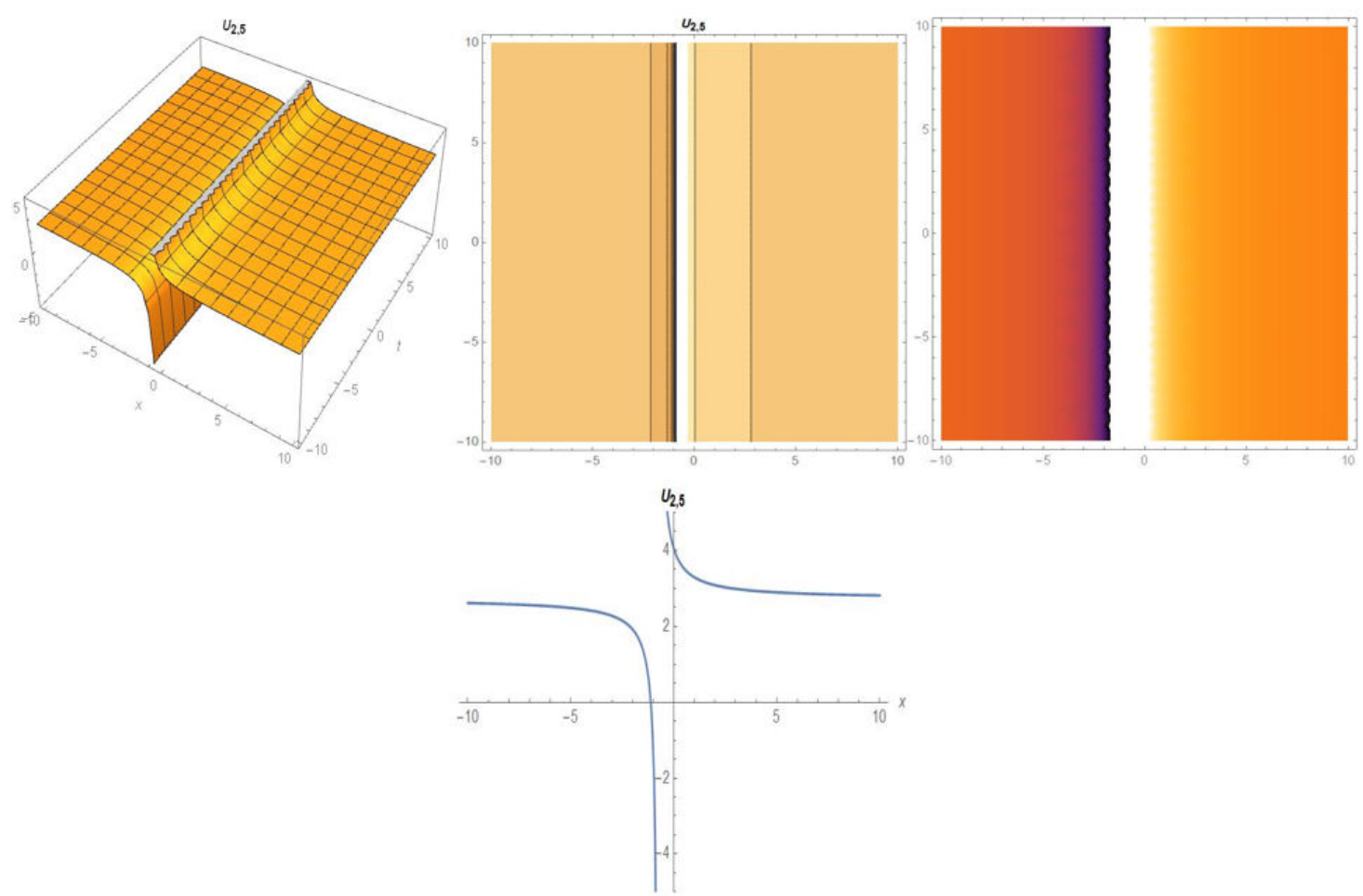

FIGURE 10. Three-dimensional, contour and density plots of the solution (40) for the values $E=0.75, a=0.2, \alpha=0.5, \lambda=1$, $c=0.860663, \sigma=0, \mu=0$, and two-dimensional $t=0.8$.

Family 4. When, $\mu \neq 0, \sigma \neq 0$ and $\sigma^{2}-4 \mu=0$

$$
u_{2,4}(x, t)=\left(\frac{1}{6}\left[3+\frac{\sqrt{36-3 a}}{\sqrt{a}}+\lambda\left\{-3+\frac{6}{(2+[E+x] \lambda)-\lambda^{2}(x-\mu)}\right\}\right]\right) .
$$

Family 5. When, $\mu=0, \sigma=0$ and $\sigma^{2}-4 \mu=0$

$$
u_{2,5}(x, t)=\left(\frac{1}{6}\left[3+\frac{\sqrt{36-3 a}}{\sqrt{a}}+\frac{6}{E+x}\right]\right) .
$$

Example 2. Let's consider the modifie Benjamin-Bona-Mahony equation with the conformable Atangana derivatives [25],

$$
{ }_{0}^{A} D_{t}^{\alpha}\{u\}+{ }_{0}^{A} D_{x}^{\alpha}\{u\}-k u_{0}^{2}{ }_{0}^{A} D_{x}^{\alpha}\{u\}+{ }_{0}^{A} D_{x}^{3 \alpha}\{u\}=0 .
$$

We reduce it to the following nonlinear ordinary differential equation form by applying the wave transformation for Eq. (41),

$$
u(x, t)=u(\eta), \quad \text { and } \quad \eta=\frac{\gamma}{\alpha}\left(x+\frac{1}{\Gamma(\alpha)}\right)^{\alpha}-\frac{\lambda}{\alpha}\left(t+\frac{1}{\Gamma(\alpha)}\right)^{\alpha},
$$

where $\lambda$ is constant. If we substitute the wave transformation (42) in Eq. (41),

$$
-\lambda u^{\prime}+\gamma u^{\prime}-k \gamma u^{2} u^{\prime}+\gamma^{3} u^{\prime \prime \prime}=0 .
$$

If Eq. (43) is integrated to simplify it,

$$
(\gamma-\lambda) u-\frac{\gamma k}{3} u^{3}+\gamma^{3}\left(u^{\prime \prime}-c\right)=0
$$

where $c$ is integral constant.

Using the balance procedure, the highest order nonlinear term $u^{3}$ and the highest order term $u^{\prime \prime}$ in Eq. (44), the following equation is get,

$$
3 n-3 m=n-m+2 \Rightarrow n=m+1
$$


If $m=1$, we then obtain $n=2$.

Accordingly, Eq. (13) can be written as follows.

$$
u(\eta)=\frac{\gamma}{\phi}=\frac{A_{0}+A_{1} e^{-\vartheta}+A_{2} e^{-2 \vartheta}}{B_{0}+B_{1} e^{-\vartheta}} .
$$

If we obtain the derivative terms required in Eq. (44) from Eq. (46),

$$
\begin{aligned}
u^{\prime}(\eta) & =\frac{\gamma^{\prime} \phi-\gamma \phi^{\prime}}{\phi^{2}}, \\
U^{\prime \prime}(\eta) & =\frac{\left(\left[\gamma^{\prime \prime} \phi^{3}+\gamma^{\prime} \phi^{\prime} \phi^{2}-\left\{\phi^{2} \gamma^{\prime} \phi^{\prime}+\phi^{2} \gamma \phi^{\prime \prime}\right\}\right]-2 \phi \phi^{\prime}\left[\gamma^{\prime} \phi-\gamma \phi^{\prime}\right]\right)}{\phi^{4}}
\end{aligned}
$$

Substituting the terms in Eqs. (46-48) into Eq. (44), we obtain exact solutions that satisfy Eq. (41). Case 3.

$$
\begin{aligned}
A_{0} & =\frac{\sqrt{\frac{3}{2}} \gamma\left(2 B_{0}^{2}-\sigma B_{0} B_{1}+2 \mu B_{1}^{2}\right)}{\sqrt{k} B_{1}} \quad A_{1}=\frac{\sqrt{\frac{3}{2}} \gamma\left(2 B_{0}+\sigma B_{1}\right)}{\sqrt{k}}, \quad A_{2}=\frac{\sqrt{6} \gamma B_{1}}{\sqrt{k}} \\
c & =\frac{2 \sqrt{6} \gamma^{4}\left(-2 B_{0}+\sigma B_{1}\right)\left(B_{0}^{2}-\sigma B_{0} B_{1}+\mu B_{1}^{2}\right)}{\sqrt{k} B_{1}^{3}}, \quad \lambda=\gamma-\frac{1}{2} \gamma^{3}\left(8 \gamma+\sigma^{2}\right)-\frac{6 \gamma^{3} B_{0}\left(B_{0}-\sigma B_{1}\right)}{B_{1}^{2}} .
\end{aligned}
$$

Substituting the coefficient obtained above into the Eq. (26), the exact solution functions of the Eq. (20) are written as follows;

Family 1. $\mu \neq 0$ and $\sigma^{2}-4 \mu>0$

$$
u_{3,1}(x, t)=\frac{\left(\left[\sqrt{\frac{3}{2}} \gamma\left\{-\sigma B_{0}+\frac{2 B_{0}^{2}}{B_{1}}+2 \mu B_{1}+\frac{8 \mu^{2} B_{1}}{\Omega^{2}}-\frac{2 \mu\left(2 B_{0}+\sigma B_{1}\right)}{\Omega}\right\}\right]\right)}{\left(\sqrt{k}\left[B_{0}-\frac{2 \mu B_{1}}{\Omega}\right]\right)}
$$
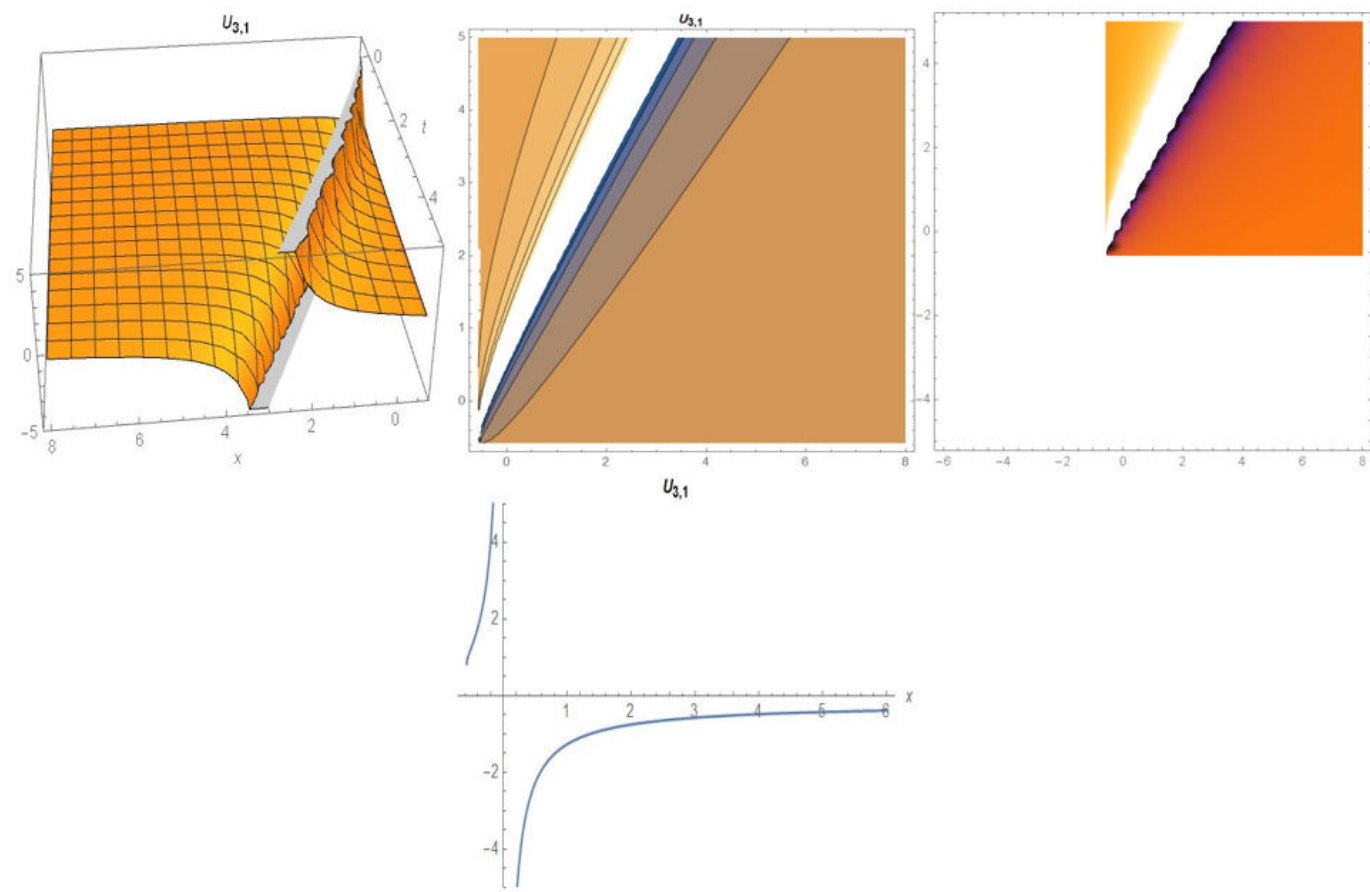

FIGURE 11. Three-dimensional, contour and density plots of the solution (50) for the values $E=0.75, \alpha=0.5, \lambda=0.186986$, $c=0.000211, \sigma=2, \mu=0, k=4, \gamma=0.2$, and two-dimensional for $t=0.8$. 
where,

$$
\Omega=\left(\sigma+\sqrt{-4 \mu+\sigma^{2}} \tanh \left[\frac{\sqrt{-4 \mu+\sigma^{2}}(E \alpha+\eta)}{2 \alpha}\right]\right)
$$

Family 2. $\mu \neq 0$ and $\sigma^{2}-4 \mu<0$

$$
u_{3,2}(x, t)=\frac{\left(\left[\sqrt{\frac{3}{2}} \gamma\left\{-\sigma B_{0}+\frac{2 B_{0}^{2}}{B_{1}}+2 \mu B_{1}+\frac{8 \mu^{2} B_{1}}{\Phi^{2}}-\frac{2 \mu\left(2 B_{0}+\sigma B_{1}\right)}{\Phi}\right\}\right]\right)}{\left(\sqrt{k}\left[B_{0}-\frac{2 \mu B_{1}}{\Phi}\right]\right)}
$$

where,

$$
\Phi=\left(\sigma-\sqrt{4 \mu-\sigma^{2}} \tan \left[\frac{\sqrt{4 \mu-\sigma^{2}}(E \alpha+\eta)}{2 \alpha}\right]\right) .
$$

Family 3. $\mu=0 \sigma \neq 0$ and $\sigma^{2}-4 \mu>0$

$$
u_{3,3}(x, t)=\left(\frac{\sqrt{\frac{3}{2}} \gamma\left[\frac{2 \lambda}{-1+e^{\sigma(E+\eta)}}-\sigma+\frac{2 B_{0}}{B_{1}}-\frac{2 \lambda\left(B_{0}-\sigma B_{1}\right)}{\left(-1+e^{\sigma(E+\eta)}\right) B_{0}+\lambda B_{1}}\right]}{\sqrt{k}}\right)
$$

Family 4. $\mu \neq 0 \sigma \neq 0$ and $\sigma^{2}-4 \mu=0$

$$
u_{3,4}(x, t)=\left(\frac{\sqrt{\frac{3}{2}} \gamma\left[-2(\lambda+\sigma) B_{0}+\frac{4 B_{0}^{2}}{B_{1}}+\left(\lambda^{2}+4 \mu-\lambda \sigma\right) B_{1}+\frac{4 \alpha^{2} \lambda^{2} B_{1}}{\nu^{2}}+\frac{2 \alpha \lambda\left\{2 B_{0}+(-2 \lambda+\sigma) B_{1}\right\}}{\nu}\right]}{\left(2 \sqrt{k}\left[B_{0}+\frac{1}{2} \lambda B_{1}\left\{-1+\frac{2 \alpha}{\nu}\right\}\right]\right)}\right)
$$
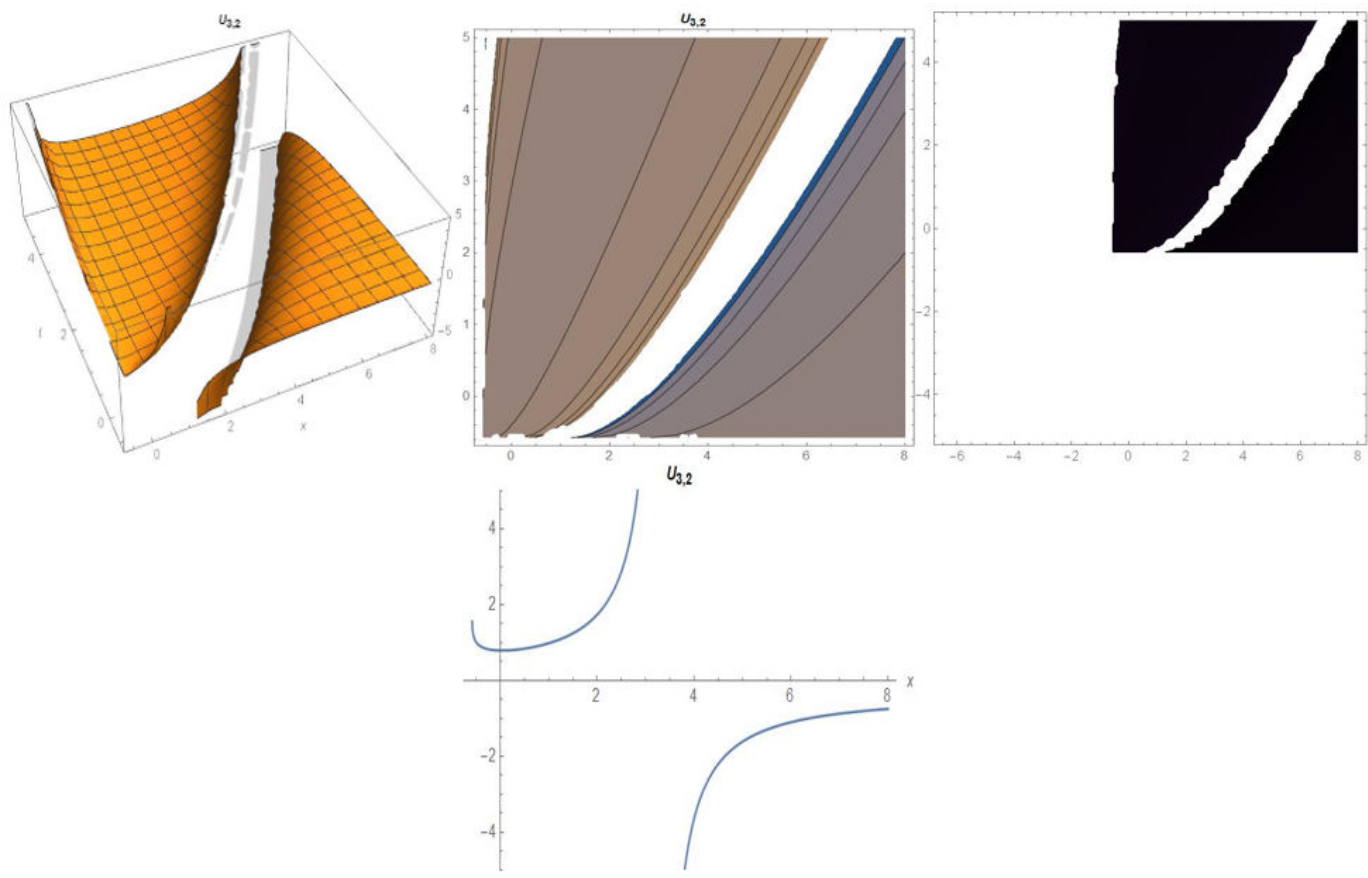

FIGURE 12. Three-dimensional, contour and density plots of the solution (51) for the values $E=0.75, \alpha=0.5, \lambda=0.186986$, $c=0.000211, \sigma=2, \mu=0, k=4, \gamma=0.2$, and two-dimensional for $t=0.8$. 

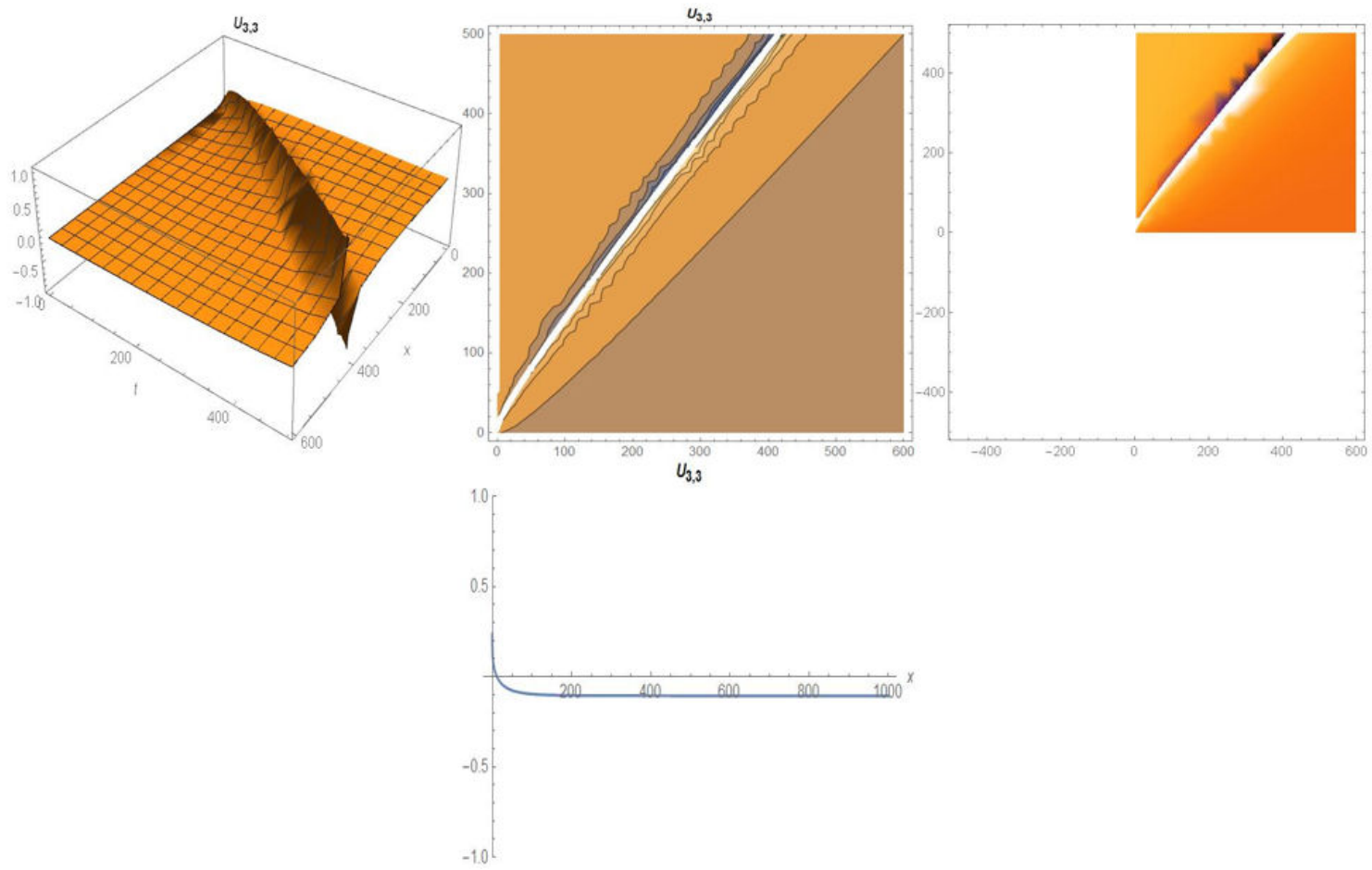

FIGURE 13. Three-dimensional, contour and density plots of the solution (52) for the values $E=0.75, \alpha=0.5, \lambda=0.198986$, $c=0.000211, \sigma=1, \mu=0, k=4, \gamma=0.2$, and two-dimensional for $t=0.8$.
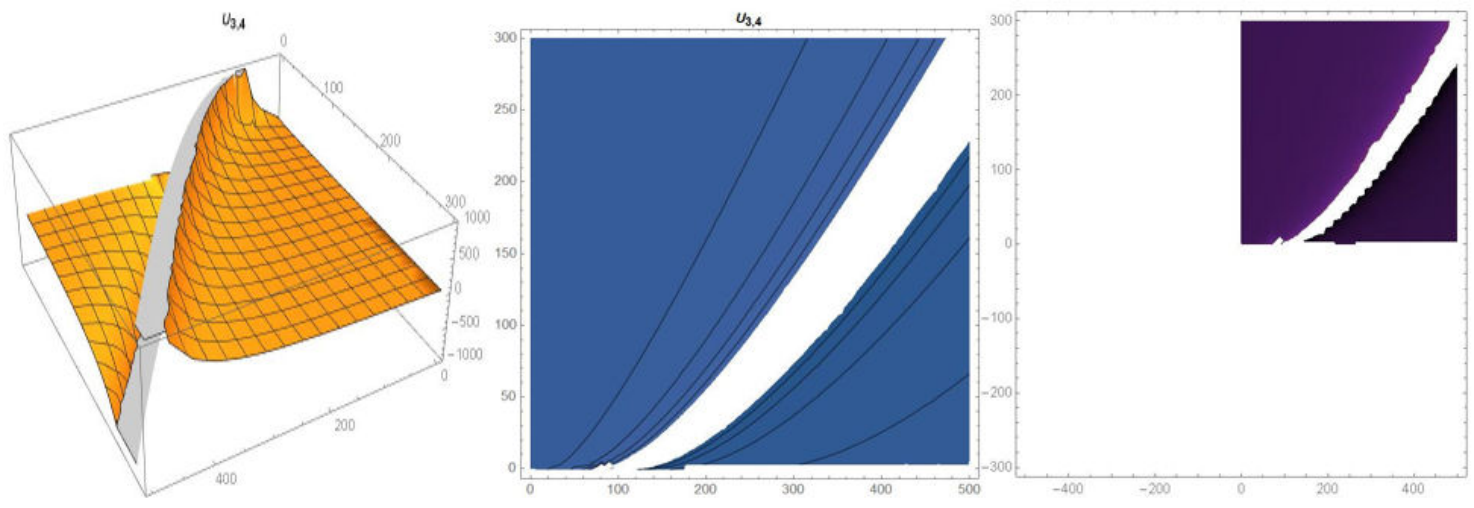

$u_{3,4}$

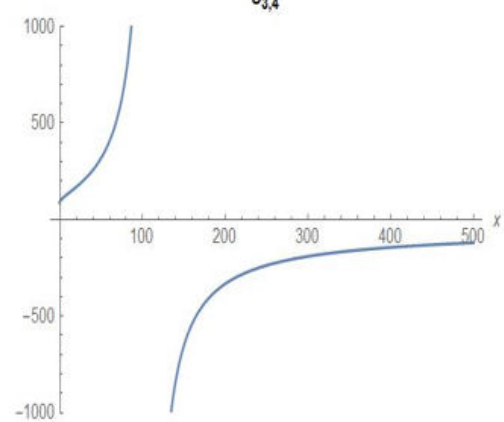

FIGURE 14. Three-dimensional, contour and density plots of the solution (53) for the values $E=0.75, \alpha=0.5, \lambda=0.153900$, $c=-0.0466588, \sigma=2, \mu=1, k=0.1, \gamma=0.2$, and two-dimensional for $t=1$. 

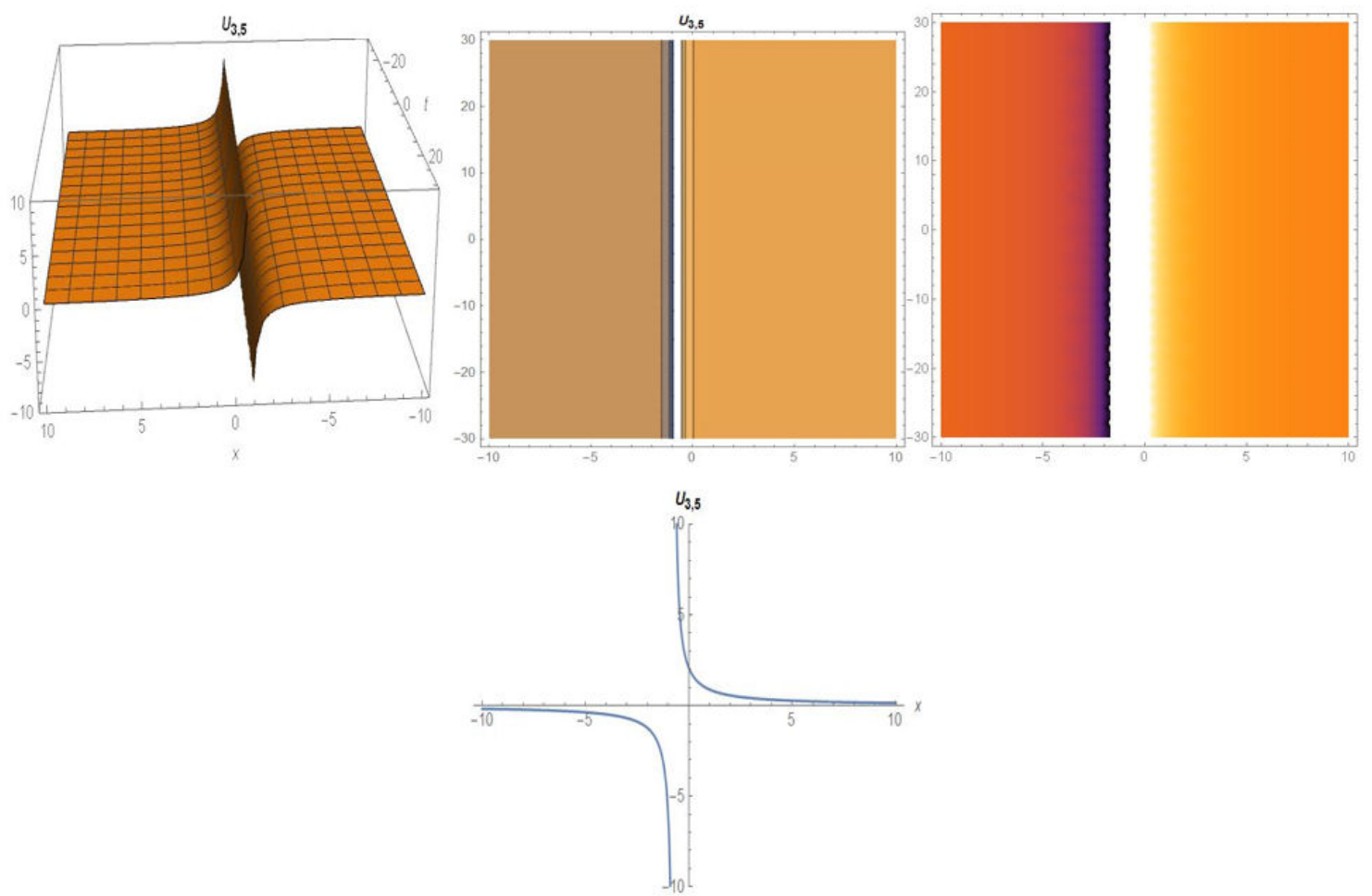

FIgURE 15. Three-dimensional, contour and density plots of the solution (54) for the values $E=0.75, \alpha=0.5, \lambda=0.199980$, $c=0.00396593, \sigma=0, \mu=0, k=0.1, \gamma=0.2$, and two-dimensional for $t=1$.

Family 5. $\mu=0 \sigma=0$ and $\sigma^{2}-4 \mu=0$

$$
u_{3,5}(x, t)=\left(\frac{\sqrt{\frac{3}{2}} \gamma\left[2 \theta^{2} B_{0}^{2}-\theta\{-2+\theta \sigma\} B_{0} B_{1}+\{2+\theta \sigma\} B_{1}^{2}\right]}{\sqrt{k} \theta B_{1}\left(B_{0}+B_{1}\right)}\right) .
$$

where $\theta=(E+x)$.

Case 4.

$$
\begin{aligned}
A_{0} & =\frac{A_{2}\left(2\left[\gamma-\lambda+\gamma^{3}\left\{2 \mu+\sigma^{2}\right\}\right] B_{0}-\sqrt{6} \sigma \sqrt{\gamma^{3}\left(\gamma-\lambda+\gamma^{3}\left[-4 \mu+\sigma^{2}\right]\right) B_{0}^{2}}\right)}{12 \gamma^{3} B_{0}} \\
A_{1} & =\sigma A_{2}-\frac{A_{2} \sqrt{\gamma^{3}\left(\gamma-\lambda+\gamma^{3}\left[-4 \mu+\sigma^{2}\right]\right) B_{0}^{2}}}{\sqrt{6} \gamma^{3} B_{0}} \\
B_{1} & =\frac{6 \gamma^{3} B_{0}^{2}}{3 \gamma^{3} \sigma B_{0}-\sqrt{6} \sqrt{\gamma^{3}\left(\gamma-\lambda+\gamma^{3}\left[-4 \mu+\sigma^{2}\right]\right) B_{0}^{2}}} \\
c & =\frac{\left(2 \gamma-2 \lambda+\gamma^{3}\left[4 \mu-\sigma^{2}\right]\right) A_{2}\left(2\left[-\gamma+\lambda+\gamma^{3}\left\{4 \mu-\sigma^{2}\right\}\right] B_{0}+\sqrt{6} \sigma \sqrt{\gamma^{3}\left(\gamma-\lambda+\gamma^{3}\left[-4 \mu+\sigma^{2}\right]\right) B_{0}^{2}}\right)}{36 \gamma^{3} B_{0}^{2}} \\
k & =-\frac{72 \gamma^{5} B_{0}\left(\left[-2 \gamma+2 \lambda+\gamma^{3}\left\{8 \mu-5 \sigma^{2}\right\}\right]\right) B_{0}-2 \sqrt{6} \sqrt{\gamma^{3}\left(\gamma-\lambda+\gamma^{3}\left[-4 \mu+\sigma^{2}\right]\right) B_{0}^{2}}}{\left(-2 \gamma+2 \lambda+\gamma^{3}\left[8 \mu+\sigma^{2}\right]\right)^{2} A_{2}^{2}}
\end{aligned}
$$

We substitute the coefficient calculated above in Eq. (26). After findin the required derivative terms in Eq. (20), we obtain the exact solution functions according to the following solution families by typing the resulting exact solution function model. 

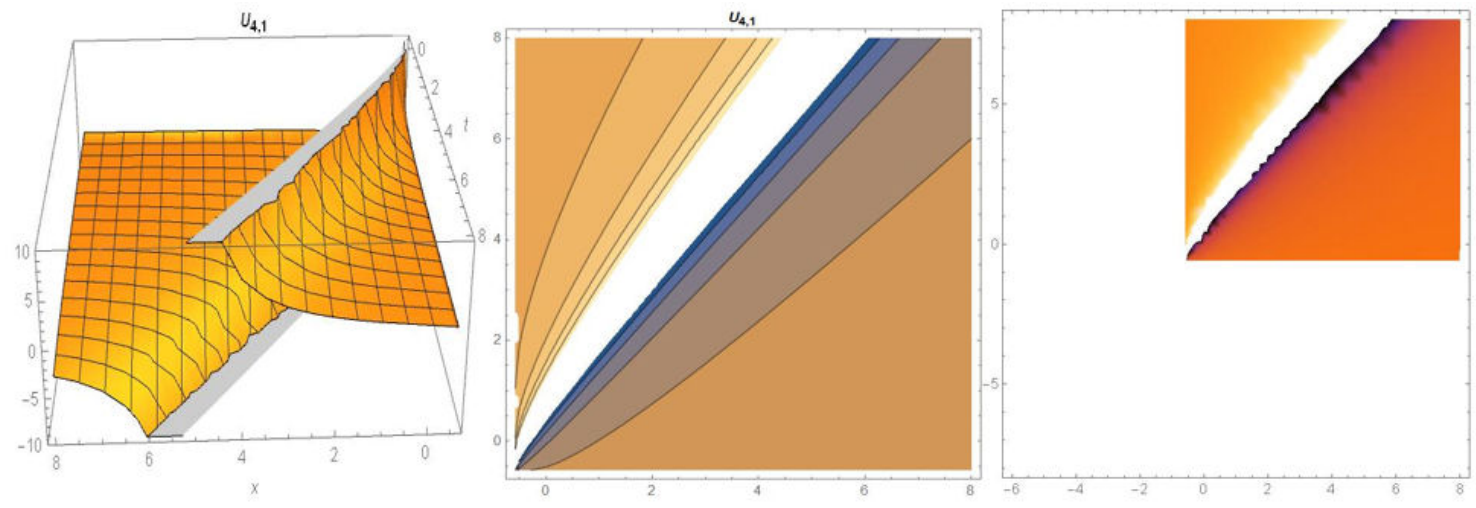

$U_{4,1}$

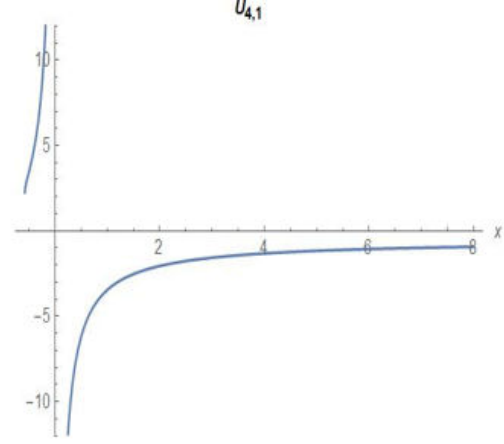

FIGURE 16. Three-dimensional, contour and density plots of the solution (56) for the values $E=0.75, \alpha=0.5, \lambda=0.186986$, $c=0.596486, \sigma=2, \mu=0.1, k=0.005894, \gamma=0.2$, and two-dimensional $t=0.8$.
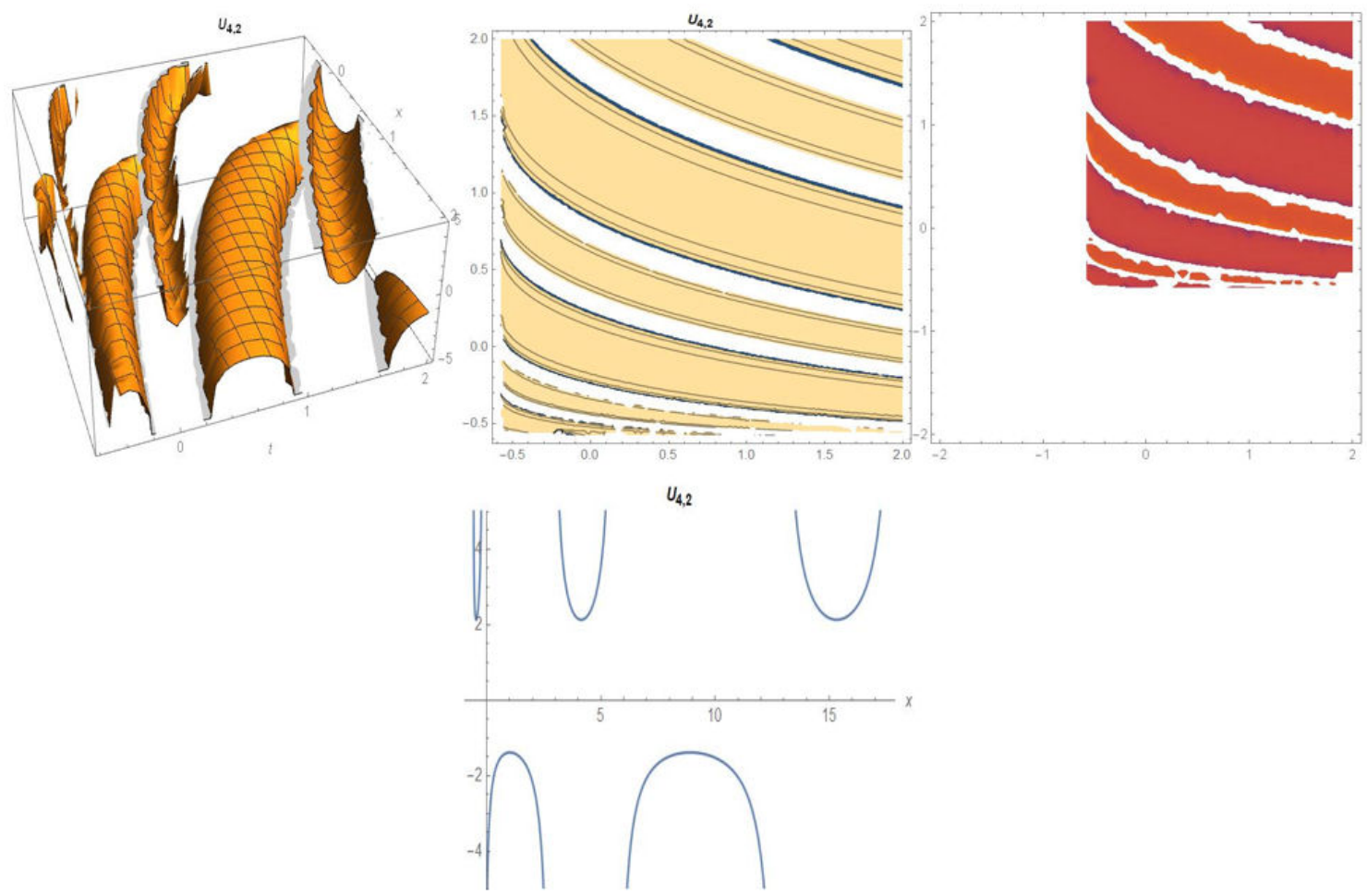

FIGURE 17. Three-dimensional, contour and density plots of the solution (57) for the values $E=0.75, \alpha=0.5, \lambda=-3, c=-1.37344$, $\sigma=1, \mu=1, k=7.127265, \gamma=1$, and two-dimensional for $t=0.8$. 
Family 1. While, $\mu \neq 0$ and $\sigma^{2}-4 \mu>0$

$$
u_{4,1}(x, t)=\frac{\frac{\left(A_{2}\left[2\left\{\gamma-\lambda+\gamma^{3}\left(2 \mu+\sigma^{2}\right)\right\} B_{0}-\sigma Z\right]\right)}{12 \gamma^{3} B_{0}}+\frac{4 \mu^{2} A_{2}}{\Lambda^{2}}+\frac{\mu A_{2}\left(-6 \gamma^{3} \sigma B_{0}+Z\right)}{3 \gamma^{3} B_{0} \Lambda}}{\left(B_{0}+\frac{12 \gamma^{3} \mu B_{0}^{2}}{-3 \gamma^{3} \sigma B_{0}+Z \Lambda}\right)},
$$

where,

$$
z=\sqrt{6\left(\gamma^{3}\left[\gamma-\lambda+\gamma^{3}\left\{-4 \mu+\sigma^{2}\right\}\right] B_{0}^{2}\right.}
$$

and

$$
\Lambda=\sigma+\sqrt{-4 \mu+\sigma^{2}} \tanh \left(\frac{1}{2 \alpha} \sqrt{-4 \mu+\sigma^{2}}(E+\eta)\right) .
$$

Family 2. While, $\mu \neq 0$ and $\sigma^{2}-4 \mu<0$

$$
u_{4,2}(x, t)=\left(\frac{\frac{\left(A_{2}\left[2\left\{\gamma-\lambda+\gamma^{3}\left(2 \mu+\sigma^{2}\right)\right\} B_{0}-\sigma Z\right]\right)}{12 \gamma^{3} B_{0}}+\frac{4 \mu^{2} A_{2}}{\Pi}+\frac{2 \mu\left[\sigma A_{2} \frac{A_{2} Z}{6 \gamma^{3} B_{0}}\right]}{-\Pi}}{\left(B_{0}+\frac{12 \gamma^{3} \mu B_{0}^{2}}{\left(-3 \gamma^{3} \sigma B_{0}+Z\right) \Pi}\right)}\right)
$$

where,

$$
\Pi=\sigma-\sqrt{4 \mu+\sigma^{2}} \tan \left(\frac{1}{2 \alpha} \sqrt{4 \mu+\sigma^{2}}(E+\eta)\right) .
$$
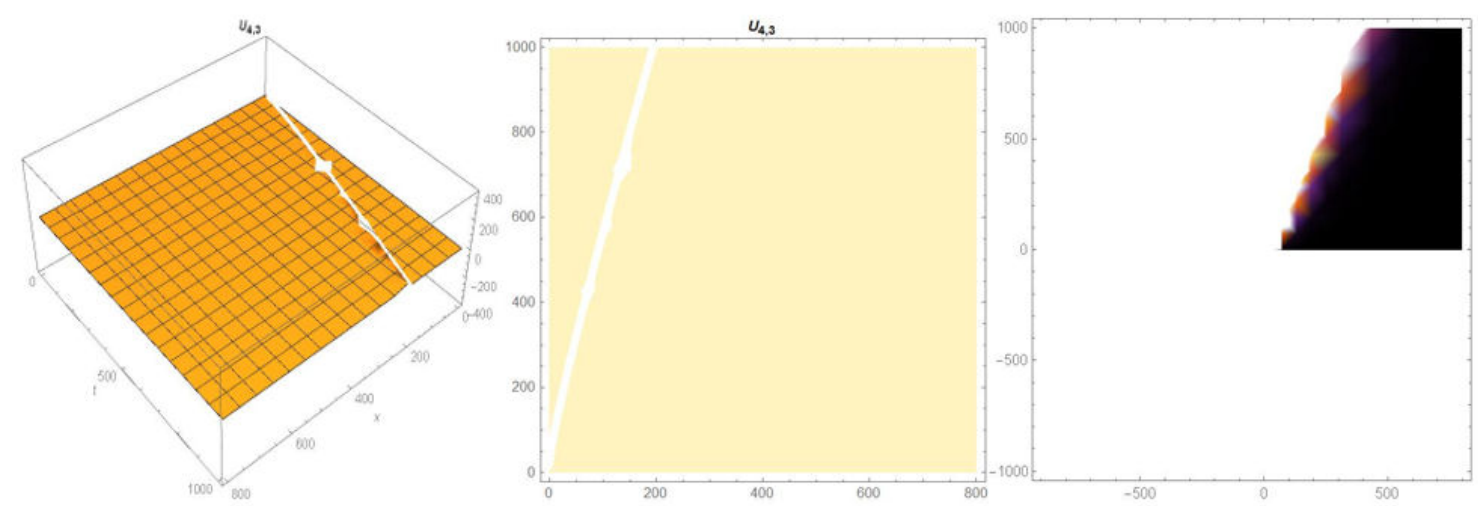

$U_{4,3}$

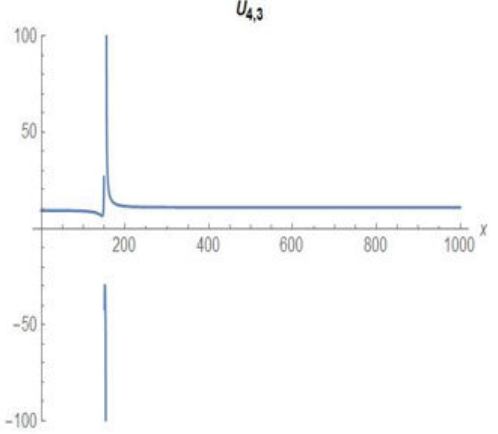

FIGURE 18. Three-dimensional, contour and density plots of the solution (58) for the values $E=0.75, \alpha=0.5, \lambda=0.1, c=-1.341331$, $\sigma=0.1, \mu=0, k=0.001235, \gamma=0.2$, and two-dimensional for $t=800$. 
Family 3. While, $\mu=0, \sigma \neq 0$ and $\sigma^{2}-4 \mu>0$

$$
u_{4,3}=\left(\frac{\frac{\lambda^{2} A_{2}}{\left[-1+e^{\sigma(E+\eta)}\right]^{2}}+\frac{A_{2}\left[2\left\{\gamma-\lambda+\gamma^{3} \sigma^{2}\right\} B_{0}-\sigma Z\right]}{12 \gamma^{3} B_{0}}+\frac{\lambda\left[\sigma A_{2}-\frac{A_{2} Z}{6 \gamma^{3} B_{0}}\right]}{\left[-1+e^{\sigma(E+\eta)}\right]}}{6 \gamma^{3} \lambda B_{0}^{2}}\right)
$$

Family 4. While, $\mu \neq 0, \sigma \neq 0$ and $\sigma^{2}-4 \mu=0$

$$
u_{4,4}=\left(\frac{A_{2}\left[2\left\{\frac{\gamma-\lambda}{\gamma^{3}}+2 \mu+\sigma^{2}\right\}-\frac{\sigma Z}{\gamma^{3} B_{0}}+\frac{\lambda^{2}\left(-6 \gamma^{3} \sigma B_{0}+Z\right)(E \alpha+\eta)}{\gamma^{3} B_{0} O}+\frac{3 \lambda^{4}(E \alpha+\eta)^{2}}{O^{2}}\right]}{\left[12\left\{B_{0}-\frac{3 \gamma^{3} \lambda^{2} B_{0}^{2}(E \alpha+\eta)}{\left(3 \gamma^{3} \sigma B_{0}-Z\right) O}\right\}\right]}\right)
$$

where $O=(\alpha[2+E \lambda]+\lambda \eta)$.

Family 5. While, $\mu=0, \sigma=0$ and $\sigma^{2}-4 \mu=0$

$$
u_{4,5}(x, t)=\left(\frac{A_{2}\left[2\left\{\frac{6}{(E+x)^{2}}+\frac{1}{\gamma^{2}}+\frac{6 \sigma}{(E+x)}+\sigma^{2}\right\}-\frac{\sqrt{6}\{2+(E+x) \sigma\} \sqrt{\gamma^{4}\left(1+\gamma^{2} \sigma^{2}\right) B_{0}}}{(E+x) \gamma^{3} B_{0}}\right]}{12\left[B_{0}-\frac{6 \gamma^{3} B_{0}^{2}}{[E+x]\left[-3 \gamma^{3} \sigma B_{0}+\sqrt{6\left(\gamma^{4}\left[1+\gamma^{2} \sigma^{2}\right] B_{0}^{2}\right)}\right]}\right]}\right)
$$
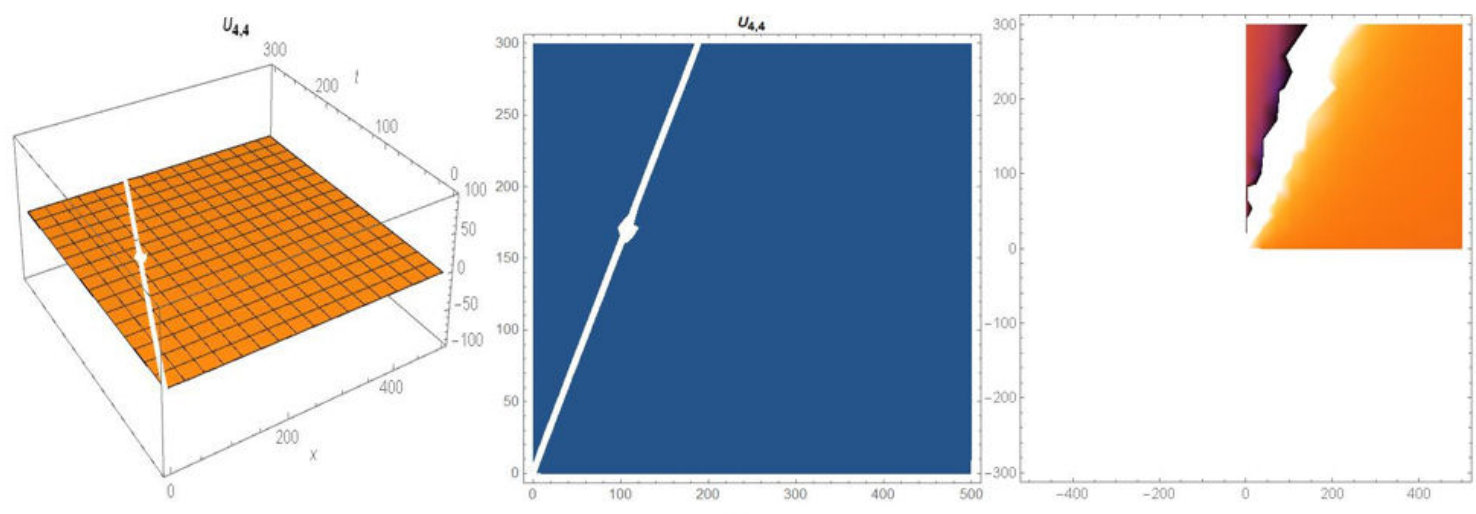

$U_{4,4}$

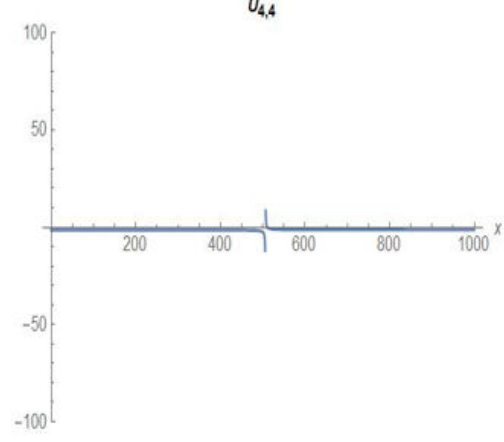

FIGURE 19. Three-dimensional, contour and density plots of the solution (59) for the values $E=0.75, \alpha=0.5, \lambda=0.1, c=(4-$ $20 \sqrt{30} / 3000), \sigma=2, \mu=1, k=-(12500(-3004-40 \sqrt{30}) / 561001), \gamma=0.2$, and two-dimensional for $t=800$. 

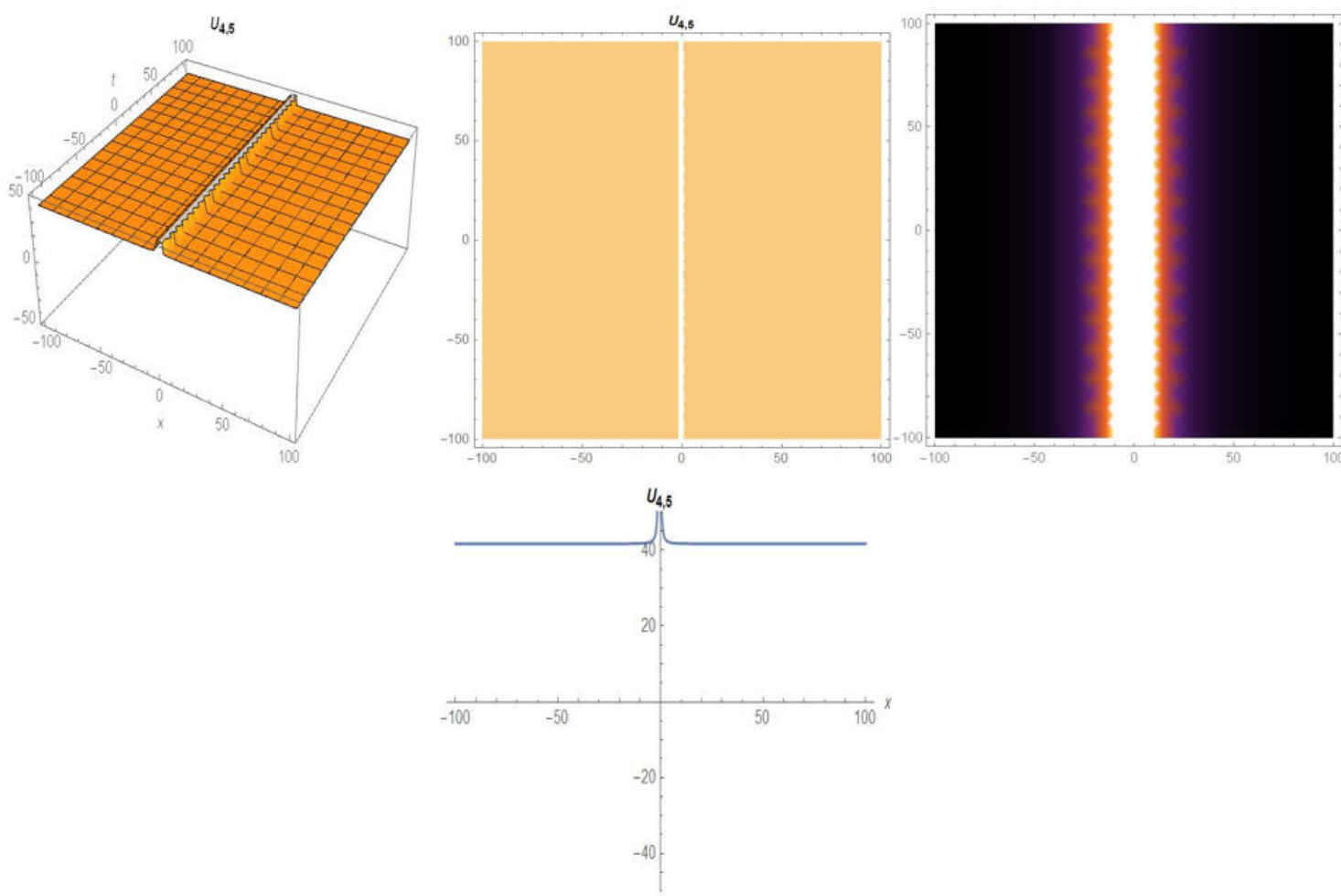

FIGURE 20. Three-dimensional, contour and density plots of the solution (40) for the values $E=0.75, \alpha=0.5, \lambda=0.1, c=1.38, \sigma=0$, $\mu=0, k=0.001152, \gamma=0.2$, and two-dimensional $t=800$.

Remark The exact solutions of the Eqs. (18) and (40) are obtained by the modifie exponential function method. Graphs representing the physical interpretations of all calculations and solution functions used during the solutions were checked and drawn using Mathematica 12. Exact solutions obtained in this study as a result of the necessary searching processes have not been found in the literature.

\section{Conclusions}

In this study, the modifie exponential function method is applied to obtain new exact solutions of the modifie BenjaminBona-Mahony and Sharma-Tasso-Olver equations given by the congruent Atangana derivative. In addition, different exact solution functions calculated for these equations are clas- sified With this method, hyperbolic and rational function solutions of the resulting functions are obtained. Parameters suitable for exact solution function models were determined and physical motion models on three-dimensional, contour, density and two-dimensional graphs were analyzed for these values. It is understood that the physical behaviors of the resulting graphics and exact solution functions have similar characters. Thus, it was concluded that the modifie exponential function method gave very effective results in findin exact solutions of other nonlinear fractional partial differential equations define by the Atangana derivative. In subsequent studies, we will investigate new exact solutions of these models by applying them to other nonlinear fractional partial differential equations define by the Atangana derivative of the modifie exponential function method.
1. C.-S. Liu, Trial equation method and its applications to nonlinear evolution equations, Acta Phys. Sinica 54 (2005) 2505, https://doi.org/10.7498/aps.54.2505

2. Y. Chen and Z. Yan, New exact solution of $(2+1)$-dimensional Gardner equation via the new sine-Gordon equation expansion method, Chaos Solitons Fractals 26 (2005) 399, https: //doi.org/10.1016/j.chaos.2005.01.004

3. N. A. Kudryashov, One method for findin exact solutions of nonlinear differential equations, Commun. Nonlinear Sci.
Numer. Simul. 17 (2012) 2248, https://doi.org/10. $1016 / j . c n s n s .2011 .10 .016$.

4. Y. Pandir, Y. Gurefe, U. Kadak, and E. Misirli, Classificatio of Exact Solutions for Some Nonlinear Partial Differential Equations with Generalized Evolution, Abstr. Appl. Anal. 2012 (2012) 478531, https://doi.org/10. $1155 / 2012 / 478531$

5. G. Shen, Y. Sun, and Y. Xiong, New Travelling-Wave Solutions for Dodd-Bullough Equation, J. Appl. Math. 2013 (2013) 364718, https://doi.org/10.1155/2013/364718 
6. H. Bulut, T. Aktürk, and Y. Gürefe, Traveling wave solutions of the $(\mathrm{N}+1)$-dimensional sine-cosine-Gordon equation, AIP Conf. Proc. 1637 (2014) 145, https : / / doi.org/10. $1063 / 1.4904573$

7. T. Aktürk, Y. Gürefe, and H. Bulut, New function method to the $(\mathrm{n}+1)$-dimensional nonlinear problems, Int. J. Optim. Control 7 (2017) 234, https://doi.org/10.11121/ijocta. 01.2017 .00489

8. S. Yunchuan, New Travelling Wave Solutions for Sine-Gordon Equation, J. Appl. Math. 2014 (2014) 841416, https:// doi.org/10.1155/2014/841416

9. I. Podlubny, Fractional Differential Equations (Academic Press, San Diego, 1999).

10. D. Kumar, J. Singh, and D. Baleanu, A hybrid computational approach for Klein-Gordon equations on Cantor sets, Nonlinear Dyn. 87 (2017) 511, https://doi.org/10.1007/ s11071-016-3057-x

11. K. M. Owolabi and A. Atangana, Numerical Simulation of Noninteger Order System in Subdiffusive, Diffusive, and Superdiffusive Scenarios, J. Comput. Nonlinear Dyn. 12 (2017) 031010, https://doi.org/10.1115/1.4035195

12. R. Metzler and J. Klafter, The random walk's guide to anomalous diffusion: a fractional dynamics approach, Phys. Rep. 339 (2000) 1, https://doi.org/10.1016/ S0370-1573(00)00070-3

13. H. M. Srivastava, D. Kumar, and J. Singh, An efficien analytical technique for fractional model of vibration equation, Appl. Math. Model. 45 (2017) 192, https://doi.org/ $10.1016 / j . a p m .2016 .12 .008$

14. H. Jafari and H. K. Kassim, Numerical solutions of telegraph and Laplace equations on Cantor sets using local fractional Laplace decomposition method, Int. J. Adv. Appl. Math. Mech. 2 (2015) 144.

15. K. M. Owolabi and A. Atangana, Numerical simulations of chaotic and complex spatiotemporal patterns in fractional reaction-diffusion systems, Comput. Appl. Math. 37 (2018) 2166, https://doi.org/10.1007/ s40314-017-0445-x

16. A. Atangana and D. Baleanu, New fractional derivatives with nonlocal and non-singular kernel: Theory and application to heat trasfer model, Therm. Sci. 20 (2016) 763, https:// doi.org/10.2298/TSCI160111018A

17. B. Lu, The firs integral method for some time fractional differential equations, J. Math. Anal. Appl. 395 (2012) 684, https : //doi.org/10.1016/j.jmaa.2012.05.066
18. X. J. Yang and Y. D. Zhang, A New Adomian Decomposition Procedure Scheme for Solving Local Fractional Volterra Integral Equation, Adv. Inf. Technol. Manag. 1 (2012) 158.

19. W. Deng, Finite Element Method for the Space and Time Fractional Fokker-Planck Equation, SIAM J. Numer. Anal. 47 (2009) 204, https://doi.org/10.1137/080714130

20. M.-S. Hu, R. P. Agarwal, and X.-J. Yang, Local Fractional Fourier Series with Application to Wave Equation in Fractal Vibrating String, Abstr. Appl. Anal. 2012 (2012) 567401, https://doi.org/10.1155/2012/567401

21. G.-H. Gao, Z.-Z. Sun, and Y.-N. Zhang, A finit difference scheme for fractional sub-diffusion equations on an unbounded domain using artificia boundary conditions, J. Comput. Phys. 231 (2012) 2865, https : / /doi.org/10.1016/j.jcp. 2011.12 .028

22. Y. Gurefe, The generalized Kudryashov method for the nonlinear fractional partial differential equations with the betaderivative, Rev. Mex. Fis. 66 (2020) 771, https://doi. org/10.31349/RevMexFis.66.771

23. M. Eslami and H. Rezazadeh, The firs integral method for Wu-Zhang system with conformable time-fractional derivative, Calcolo 53 (2016) 475, https://doi.org/10.1007/ s10092-015-0158-8

24. G. Yel and H. M. Baskonus, Solitons in conformable time-fractional Wu-Zhang system arising in coastal design, Pramana 93 (2019) 57, https://doi.org/10.1007/ s12043-019-1818-z

25. R. Khalil, M. Al Horani, A. Yousef, and M. Sababheh, A new definitio of fractional derivative, J. Comput. Appl. Math. 264 (2014) 65, https://doi.org/10.1016/j.cam. 2014.01 .002

26. A. Atangana, D. Baleanu, and A. Alsaedi, New properties of conformable derivative, Open Math. 13 (2015) 889, https: //doi.org/10.1515/math-2015-0081

27. H. Yépez-Martínez, J. F. Gómez-Aguilar, and A. Atangana, First integral method for non-linear differential equations with conformable derivative, Math. Model. Nat. Phenom. 13 (2018) 14, https://doi.org/10.1051/mmnp/2018012.

28. H.-O. Roshid, and M. A. Rahman, The $\exp (-\Phi(\eta))$-expansion method with application in the (1+1)-dimensional classical Boussinesq equations, Results Phys. 4 (2014) 150, https: //doi.org/10.1016/j.rinp.2014.07.006 